\title{
EIGENFUNCTION EXPANSIONS ASSOCIATED WITH THE LAPLACIAN FOR CERTAIN DOMAINS WITH INFINITE BOUNDARIES. I( $\left.{ }^{1}\right)$
}

\author{
BY \\ CHARLES IRWIN GOLDSTEIN
}

1. Introduction. In this paper we shall consider the selfadjoint operator $A$ given by the negative Laplacian acting on functions that satisfy certain homogeneous boundary conditions in a class of domains with infinite boundaries in $N$-dimensional Euclidean space, $R^{N}(N \geqq 2)$. We shall construct two complete, orthogonal sets of generalized eigenfunctions for the operator $A$. These generalized eigenfunctions will be referred to as distorted plane waves. In a succeeding paper we shall apply these results to scattering theory.

Distorted plane waves were first used in proving an expansion theorem by Ikebe [6]. He treated the case of the Schroedinger operator $-\Delta+q(x)$ acting on $L_{2}\left(R^{3}\right)$, $q(x)$ being a potential function. Distorted plane waves were also used by Shenk [13] and Shizuta [14] in dealing with the operator $-\Delta$ acting on $L_{2}(D), D$ being the exterior of a bounded domain with smooth boundary. In [13], it was proven that for $D \subset R^{N}(N \geqq 2)$, this operator is unitarily equivalent to the operator $-\Delta$ acting on $L_{2}\left(R^{N}\right)$. This result was also proven in a paper by Lax and Phillips [9] (for $N$ odd), employing a different method.

The domains $\Omega$ we are considering are perturbed infinite or semi-infinite cylinders. $\Omega$ is contained in an infinite (or semi-infinite) cylinder $S$ and obtained from $S$ by perturbing a finite portion of the boundary of $S$. The cylinder $S$ may have arbitrary cross-section.

We first consider the operator $A_{0}(A)$ given by $-\Delta$ acting on functions which vanish on the boundary $\dot{S}(\dot{\Omega})$ of $S(\Omega)$. Separation of variables yields a complete, orthogonal set of generalized eigenfunctions $W_{n}^{0}(x ; \lambda)$ for the operator $A_{0}$. These functions satisfy the boundary value problem:

$$
(\Delta+\lambda) W_{n}^{0}(x ; \lambda)=0 \text { in } S, \quad W_{n}^{0}(x ; \lambda)=0 \text { on } \dot{S} .
$$

In $\S 2$, we use these generalized eigenfunctions in the usual manner to establish a spectral representation for the operator $A_{0}$. We easily conclude from this representation that the spectrum of $A_{0}, \sigma\left(A_{0}\right)$, is absolutely continuous and $m_{0}(\lambda)$, the spectral multiplicity of each point $\lambda$ in $\sigma\left(A_{0}\right)$ is finite, piecewise constant and nondecreasing. Furthermore $m_{0}(\lambda) \rightarrow \infty$ as $\lambda \rightarrow \infty$.

Received by the editors August 6, 1967.

(1) Part of this work was performed under the auspices of the U. S. Atomic Energy Commission and contract AT(30-1)-1480 at the Courant Institute of Mathematical Sciences, New York University, New York. 
In $\S 3-5$, we show, using the method of distorted plane waves that for a perturbed infinite cylinder $\Omega$, the continuous part of the operator $A$, denoted by $A^{c}$, is unitarily equivalent to $A_{0}$. In $\S 3$ we construct a set of generalized eigenfunctions $W_{n}^{ \pm}(x ; \lambda)$ for the operator $A$. They are expressed in the form $W_{n}^{ \pm}(x ; \lambda)=W_{n}^{0}(x ; \lambda)$ $+V_{n}^{ \pm}(x ; \lambda) . V_{n}^{+}(x ; \lambda)$, the "outgoing solutions", satisfy the boundary value problem

$$
(\Delta+\lambda) V_{n}^{+}(x ; \lambda)=0 \text { in } \Omega, \quad V_{n}^{+}(x ; \lambda)=-W_{n}^{0}(x ; \lambda) \text { on } \dot{\Omega},
$$

as well as certain "radiation conditions" at infinity. These conditions correspond to the outgoing Sommerfeld radiation conditions associated with the exterior problem. The functions $V_{n}^{+}(x ; \lambda)$ are constructed with the aid of the principle of limiting absorption, proven by Eidus [3] for a semi-infinite cylinder. A second set of generalized eigenfunctions $W_{n}^{-}(x ; \lambda)=W_{n}^{0}(x ; \lambda)+V_{n}^{-}(x ; \lambda)$ may be constructed in the same way. Here $V_{n}^{-}(x ; \lambda)$ satisfies certain "incoming radiation conditions". The methods of potential theory used in [13] and [14] to establish the existence of the distorted plane waves are not applicable here since the boundary of $\Omega$ has infinite length.

In $\$ \$ 4$ and 5 we construct a pair of spectral representations for the operator $A^{c}$, using the generalized eigenfunctions $W_{n}^{ \pm}(x ; \lambda)$. The main results of the paper are embodied in Theorems 4.1 and 5.1. Lemma 4.2, which enables us to treat the case $N \geqq 4$, is analogous to a result proven in [13].

In $\$ 6$, we discuss the perturbed semi-infinite cylinder, proving all of the above results for this case. In particular it follows that for a class of perturbed semiinfiite cylinders $\Omega$, considered by Rellich [11], for which $A$ has no point eigenvalues, $A$ is unitarily equivalent to $A_{0}$.

In $§ 7$, we consider the more general class of boundary conditions

$$
\frac{\partial \mu\left(x_{1}, \ldots, x_{N}\right)}{\partial n}=\rho\left(x_{1}, \ldots, x_{N-1}\right) \mu\left(x_{1}, \ldots, x_{N}\right)
$$

where $\rho\left(x_{1}, \ldots, x_{N-1}\right) \leqq 0$ and $\rho$ is a piecewise continuous function. Results analogous to those of $\S \S 3$ and 4 are given for the operators $A_{0}^{\rho}$ and $A^{\rho}$ determined by these boundary conditions.

Finally in $\S 8$ we consider a class of perturbed infinite (semi-infinite) cylinders $\Omega$, which need not satisfy the condition $\Omega \subseteq S$. In this case it is shown that there can be at most a finite number of bounded linearly independent generalized eigenfunctions associated with any real number $\lambda$. The proof is based on a generalized Phragmén-Lindelöf Theorem, due to Lax [8], dealing with the solutions of elliptic boundary value problems in a perturbed cylinder.

2. Preliminary results. The main result of this section is an expansion theorem, dealing with functions square integrable in a cylinder. We begin with some definitions. 
Suppose $B$ is an arbitrary domain in $R^{N}$ with boundary $\dot{B}$. Set $x=\left(x_{1}, \ldots, x_{N}\right)$, $\tilde{x}=\left(x_{1}, \ldots, x_{N-1}\right)$. Thus $x=\left(\tilde{x}, x_{N}\right)$. For each function $\varphi(x)$ in $C_{0}^{\infty}(B)$, and each integer $m \geqq 0$, set

$$
\|\varphi\|_{m_{(B)}}=\left(\sum_{|\alpha| \leqq m} \int_{B}\left|D^{\alpha} \varphi\right|^{2} d x\right)^{1 / 2},
$$

where $\alpha=\left(\alpha_{1}, \ldots, \alpha_{N}\right),|\alpha|=\sum_{j=1}^{N} \alpha_{j}$, each $\alpha_{j}$ is a nonnegative integer, and

$$
D^{\alpha} \varphi=\frac{\partial^{|\alpha|} \varphi(x)}{\partial x_{1}^{\alpha}{ }^{1} \cdots \partial x_{N}^{\alpha_{N}}}
$$

We denote by $\dot{H}_{m}(B)$ the closure of $C_{0}^{\infty}(B)$ under the norm given by \|\|$_{m_{(B)}}$.

We consider the Friedrichs extension, $A_{B}$, of the operator given by $-\Delta$ acting on $C_{0}^{\infty}(B) . A_{B}$ may be defined as follows. The domain of $A_{B}, D\left(A_{B}\right)$, shall consist of all functions $\mu(x)$ in $\dot{H}_{1}(B)$ for which there exists a function $w(x)$ in $\dot{H}_{0}(B)=L_{2}(B)$ satisfying the condition:

$$
\sum_{j=1}^{N} \int_{B} \frac{\partial \varphi}{\partial x_{j}} \frac{\partial \bar{\mu}}{\partial x_{j}} d x=\int_{B} \varphi \bar{w} d x
$$

for all $\varphi(x)$ in $\dot{H}_{1}(B)$. For each such $\mu(x)$, set $A_{B} \mu=w$. It is well known [4] that the operator $A_{B}$ is nonnegative.

Denote by $S^{\prime}$ the semi-infinite cylinder $x_{N} \geqq 0, \tilde{x} \in l$, where $l$ is a bounded $N-1$ dimensional domain lying in the hyperplane $x_{N}=0$. We next obtain a spectral representation for the operator $A_{S^{\prime}}$. Consider the set of elements of the form $f=\left\{f_{n}(\xi)\right\}=\left\{f_{1}(\xi), f_{2}(\xi), \ldots\right\}$, where each function $f_{n}(\xi)$ is square integrable over $(0, \infty)$, and $\sum_{n=1}^{\infty} \int_{0}^{\infty}\left|f_{n}(\xi)\right|^{2} d \xi<\infty$. For any two such elements $f=\left\{f_{n}(\xi)\right\}$, $q=\left\{q_{n}(\xi)\right\}$, set

$$
(f, q)_{H}=\sum_{n=1}^{\infty} \int_{0}^{\infty} f_{n}(\xi) \bar{q}_{n}(\xi) d \xi .
$$

Denote the resulting Hilbert space by $H$.

Since the cylindrical cross-section $l$ is a bounded $N-1$ dimensional domain, it follows that there exists a complete, countable, orthonormal set of eigenfunctions for the operator $A_{l}$. Denote the eigenvalues of $A_{l}$, ordered increasingly, by $\left\{v_{n}\right\}$ and the corresponding orthonormal eigenfunctions by $\eta_{n}(\tilde{x})$. For each $f(x)$ in $L_{2}\left(S^{\prime}\right)$, set

$$
T_{0} f=\operatorname{lim.m.~}_{x_{N} \rightarrow \infty}\left\{\left(\frac{2}{\pi}\right)^{1 / 2} \int_{l} \int_{0}^{x_{N}} \sin \xi x_{N} \bar{\eta}_{N}(\tilde{x})\right\} f(x) d x,
$$

where l.i.m. signifies that the limit is to be taken in the sense of the Hilbert space $H$. Denote $T_{0} f$ by $\hat{f}^{0}=\left\{\hat{f}_{n}^{0}(\xi)\right\}$.

For each element $q=\left\{q_{n}(\xi)\right\}$ in $H$, set

$$
T_{1} q=\left(\frac{2}{\pi}\right)^{1 / 2} \underset{M \rightarrow \infty ; \xi^{\bullet} \rightarrow \infty}{\lim } \sum_{n=1}^{M} \int_{0}^{\xi *} q_{n}(\xi) \sin \xi x_{N} \eta_{n}(\tilde{x}) d \xi,
$$


where the limit is to be taken in the sense of $L_{2}\left(S^{\prime}\right)$. We now state without proof the expansion theorem referred to at the beginning of this section. It may be proven in the same way as the ordinary Fourier expansion theorem for functions in $L_{2}\left(R^{N}\right)$.

THEOREM 2.1. $T_{0}$ is a unitary transformation from $L_{2}\left(S^{\prime}\right)$ onto $H . T_{1}=T_{0}^{*}=T_{0}^{-1}$.

The functions $w_{n}^{0}(x ; \xi)=(2 / \pi)^{1 / 2} \sin \xi x_{N} \eta_{n}(\tilde{x})$ constitute a set of generalized eigenfunctions of the operator $A_{S^{\prime}} . w_{n}^{0}(x ; \xi)$ satisfies the boundary value problem

$$
\left(\Delta+\xi^{2}+v_{n}\right) w_{n}^{0}(x ; \xi)=0, \quad w_{n}^{0}(x ; \xi)=0 \text { on } \dot{S}^{\prime}
$$

The functions $w_{n}^{0}(x ; \xi)$ will be referred to as plane waves. Theorem 2.1 tells us that they form a complete, orthogonal set of generalized eigenfunctions of $A_{S^{\prime}}$. For each function $\varphi(x)$ in $C_{0}^{\infty}\left(S^{\prime}\right)$, it follows by integration by parts that

$$
T_{0}(-\Delta \varphi)=\left\{\left(\xi^{2}+v_{n}\right) \hat{\varphi}_{n}^{0}(\xi)\right\} \text {. }
$$

For each $n=1,2, \ldots$, make the substitution $\lambda=\xi^{2}+\nu_{n}$. Set $U_{0} f=\left\{\hat{f}_{n}^{0}\left(\left(\lambda-\nu_{n}\right)^{1 / 2}\right)\right\}$ $=\left\{q_{n}(\lambda)\right\}$ for every function $f(x)$ in $L_{2}\left(S^{\prime}\right)$. By Theorem 2.1, we have:

$$
\|f\|_{L_{2}\left(S^{\prime}\right)}^{2}=\left\|\hat{f}^{0}\right\|_{H}^{2}=\sum_{n=1}^{\infty} \int_{v_{n}}^{\infty}\left|q_{n}(\lambda)\right|^{2} \frac{d \lambda}{2\left(\lambda-\nu_{n}\right)^{1 / 2}}
$$

Set $H^{\prime}=\bigoplus_{n=1}^{\infty} L_{2}\left(v_{n}, \infty ; d \lambda / 2\left(\lambda-v_{n}\right)^{1 / 2}\right)$. It follows that $U_{0}$ is a unitary mapping from $L_{2}\left(S^{\prime}\right)$ onto $H^{\prime}$. We also have $U_{0}\left(A_{S^{\prime}} f\right)=\left\{\lambda q_{n}(\lambda)\right\}$ for each $f$ in $D\left(A_{S^{\prime}}\right)$. Thus $U_{0}$ gives a spectral representation of the selfadjoint operator $A_{S^{\prime}}$. The spectrum of $A_{S^{\prime}}$ is continuous and the spectral multiplicity $m_{0}(\lambda)$ has the value $n$ for $\nu_{n} \leqq \lambda<\nu_{n+1}$. Thus $m_{0}(\lambda)$ jumps at each point $\nu_{n}$ and $m_{0}(\lambda) \rightarrow \infty$ as $\lambda \rightarrow \infty$.

Analogous results hold for the operator $A_{S}$ where $S$ is an infinite cylinder, with cross-section $l$. In this case, the generalized eigenfunctions $w_{n}^{0}(x ; \xi)$ are given by $(2 \pi)^{-1 / 2} e^{i \xi x_{N}} \eta_{n}(\tilde{x})$, where $n=1,2, \ldots$, and $\xi \in R^{1}$. The completeness and orthogonality of these generalized eigenfunctions as well as the spectral representation of $A_{S}$ follows in the same way.

3. The distorted plane waves. We begin this section by defining precisely the domains $\Omega$ we shall be considering. Let $\Omega$ be a domain in $R^{N}$ composed of two parts $\Omega_{1}$ and $\Omega_{2}$. Suppose that $\Omega$ satisfies the following conditions (see Figure 1).

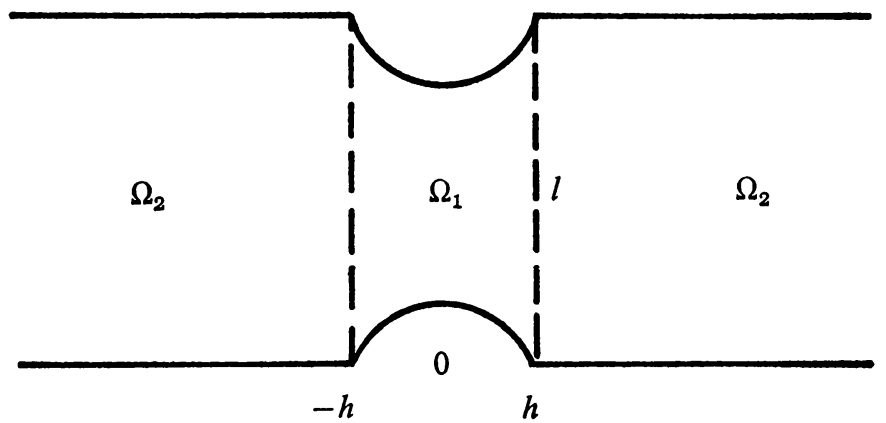

FIGURE 1 
(1) $\Omega$ is contained in the infinite cylinder $S$ described in $\S 1$.

(2) $\Omega_{1}$ is bounded and is contained in $-h \leqq x_{N} \leqq h(0<h)$.

(3) $\Omega_{2}$ coincides with $S$ for $h \leqq\left|x_{N}\right|$.

(4) $\dot{\Omega}$ is a $C^{\infty} \operatorname{surface}\left({ }^{2}\right)$.

Let $\Omega_{\dot{x}_{N}}\left(\dot{\Omega}_{\dot{x}_{N}}^{\prime}\right)$ represent that part of $\Omega(\dot{\Omega})$ in which $\left|x_{N}\right| \leqq \dot{x}_{N}$. Let $\Omega_{x_{N}^{1} x_{N}^{2}}\left(\dot{\Omega}_{x_{N}^{\prime} x_{N}^{2}}^{\prime}\right)$ represent that part of $\Omega(\dot{\Omega})$ in which $x_{N}^{1} \leqq x_{N} \leqq x_{N}^{2}$. Denote

$$
\|f\|_{m_{\left(\Omega_{\dot{x}_{N}}\right.}} \text { by }\|f\|_{m_{\left(\dot{x}_{N}\right)}}
$$

for each $f$ in $\dot{H}_{m}\left(\Omega_{\dot{x}_{N}}\right)$, with a similar notation for

$$
\|f\|_{m_{\left(\Omega_{x_{N} x_{N}^{2}}^{2}\right.}}
$$

Finally, let $l_{\dot{x}_{N}}$ represent the intersection of $\Omega$ with the hyperplane $x_{N}=\dot{x}_{N}$.

Denote $A_{S}$ and $A_{\Omega}$ by $A_{0}$ and $A$, respectively. $A$ may have at most a countable number of point eigenvalues $\left\{\lambda_{j}\right\}$ of finite multiplicity $\left({ }^{3}\right)$. Denoting the orthonormal eigenfunctions of $A$ by $\left\{w_{j}(x)\right\}$, we let $P$ denote the projection operator taking $L_{2}(\Omega)$ onto the subspace spanned by the eigenfunctions $\left\{w_{f}(x)\right\}$. Set $A^{c}=(I-P) A$. Thus $A^{c}$ represents the continuous part of the operator $A$. We shall ultimately prove that $A^{c}$ is unitarily equivalent to $A_{0}$.

In the remainder of this section we shall construct a set of generalized eigenfunctions (distorted plane waves) of the operator $A$. We begin by solving the following boundary value problem:

$$
(-\Delta-\lambda) \mu(x)=F(x) \text { in } \Omega, \quad \mu(x)=0 \text { on } \dot{\Omega},
$$

where $\lambda>0, F(x) \in C^{\infty}(\Omega)$, and $F(x) \equiv 0$ for $h<h^{\prime} \leqq\left|x_{N}\right|$. We proceed to solve this boundary value problem, using the method of limiting absorption employed by Eidus [3] in dealing with a perturbed semi-infinite cylinder.

For $\lambda \in \sigma(A)$, (3.1) will not always have a solution in $L_{2}(\Omega)$. We first define a topological space $\chi$ in which (3.1) is a well-posed problem. Consider the set of functions $f(x)$ defined in $\Omega$, which satisfy the following conditions:

(a) $f \in H_{2}^{\text {loc }}(\Omega)$. (This means that for each compact interior subregion $\Omega^{\prime} \subset \Omega$, we have $f \in H_{2}\left(\Omega^{\prime}\right)$, i.e., $D^{\alpha} f \in L_{2}\left(\Omega^{\prime}\right)$ for $|\alpha| \leqq 2$. By definition

$$
\left.\|f\|_{2_{\left(\Omega^{\prime}\right)}^{2}}^{2}=\sum_{|\alpha| \leqq 2} \int_{\Omega^{\prime}}\left|D^{\alpha}\right|^{2} d x .\right)
$$

(b) There exists a sequence of functions $\left\{f_{n}(x)\right\}$ such that $f_{n} \in H_{1}^{\text {loc }}(\Omega), f_{n}(x)$ vanishes in boundary strips along $\dot{\Omega}$, and such that in any region $\Omega_{\dot{x}_{N}}$, we have $\left\|f_{n}-f\right\|_{1_{\left(\dot{x}_{N}\right)}} \rightarrow 0$. Denote the set of functions satisfying (a) and (b) by $\chi^{\prime}$. We define

$\left({ }^{2}\right)$ Actually we need only assume that $\dot{\Omega}$ belongs to class $C^{N+2}$.

$\left({ }^{3}\right)$ The finite multiplicity follows from Theorem 8.1 . 
a topology in the set $\chi^{\prime}$ as follows. A sequence $\left\{f_{n}\right\}$ in $\chi^{\prime}$ will be said to converge to a function $f(x)$ (written $f_{n} \vec{x} f$ ) if:

(a) $\left\|f_{n}-f\right\|_{2_{\left(\Omega^{\prime}\right)}} \rightarrow 0$ for each compact interior subregion $\Omega^{\prime}$, and

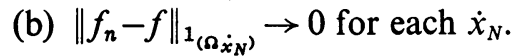

Notice that this implies $f \in \chi^{\prime}$. We denote the resulting topological space by $\chi$. For $f$ in $\chi$, set $\bar{A} f=-\Delta f$.

Let $\Lambda$ denote all those real numbers contained in $\left(\nu_{1}, \infty\right)$, other than the sequence $\left\{\nu_{n}\right\},\left\{\lambda_{n}\right\}$. For each function $\mu(x)$ in $\chi$, set

$$
\mu_{j}^{(+)}\left(x_{N}\right)=\int_{l_{X_{N}}} \mu(x) \eta_{j}(\tilde{x}) d \tilde{x} \quad \text { for } h^{\prime} \leqq x_{N}
$$

and set

$$
\mu_{j}^{(-)}\left(x_{N}\right)=\int_{l_{X_{N}}} \mu(x) \eta_{j}(\tilde{x}) d \tilde{x} \text { for } x_{N} \leqq-h^{\prime} .
$$

Now for $\lambda$ in $\Lambda$ and $\varepsilon>0$, set $\mu^{\varepsilon}(x)=(A-\lambda-i \varepsilon)^{-1} F(x)$. We shall show that as $\varepsilon \downarrow 0, \mu^{\varepsilon} \underset{x}{\longrightarrow}$, where $\mu$ satisfies (3.1) as well as the following conditions:

$$
\begin{aligned}
\mu_{j}^{( \pm)}\left(x_{N}\right) & =C_{j}^{( \pm)} \exp \left[-\left(\nu_{j}-\lambda\right)^{1 / 2}\left|x_{N}\right|\right] \\
\left(\frac{\partial \mu}{\partial x_{N}}\right)_{j}^{( \pm)}\left(x_{N}\right) & =\mp C_{j}^{( \pm)}\left(\nu_{j}-\lambda\right)^{1 / 2} \exp \left[-\left(\nu_{j}-\lambda\right)^{1 / 2}\left|x_{N}\right|\right]
\end{aligned}
$$

where $C_{j}^{( \pm)}$are constants, $\left(\nu_{j}-\lambda\right)^{1 / 2}=-i\left(\lambda-v_{j}\right)^{1 / 2}$, and $h^{\prime} \leqq\left|x_{N}\right|$. Thus $\mu(x)$ and $\partial \mu / \partial x_{N}$ will be expressed as Fourier series:

$$
\begin{aligned}
\mu(x) & =\sum_{j=1}^{\infty} C_{j}^{+} \exp \left[-\left(\nu_{j}-\lambda\right)^{1 / 2} x_{N}\right] \eta_{j}(\tilde{x}), \\
\frac{\partial \mu}{\partial x_{N}} & =\sum_{j=1}^{\infty}-C_{j}^{+}\left(\nu_{j}-\lambda\right)^{1 / 2} \exp \left[-\left(\nu_{j}-\lambda\right)^{1 / 2} x_{N}\right] \eta_{j}(\tilde{x}),
\end{aligned}
$$

for $h^{\prime} \leqq x_{N}$, with a similar expansion for $x_{N} \leqq-h^{\prime}$.

LEMMA 3.1 .

$$
\begin{aligned}
\left(\mu^{\varepsilon}\right)_{j}^{( \pm)}\left(x_{N}\right) & =C_{j}^{\varepsilon( \pm)} \exp \left[-\left(\nu_{j}-\lambda-i \varepsilon\right)^{1 / 2}\left|x_{N}\right|\right], \\
\left(\frac{\partial \mu^{\varepsilon}}{\partial x_{N}}\right)_{j}^{( \pm)}\left(x_{N}\right) & =\mp C_{j}^{\varepsilon( \pm)}\left(\nu_{j}-\lambda-i \varepsilon\right)^{1 / 2} \exp \left[-\left(\nu_{j}-\lambda-i \varepsilon\right)^{1 / 2}\left|x_{N}\right|\right],
\end{aligned}
$$

where $\operatorname{Re}\left(\nu_{j}-\lambda-i \varepsilon\right)^{1 / 2}>0$, the $C_{j}^{\varepsilon( \pm)}$ are constants, and $h^{\prime} \leqq\left|x_{N}\right|$.

To prove the lemma, we observe that: (1) the functions $\left(\mu^{\varepsilon}\right)_{j}^{( \pm)}\left(x_{N}\right)$ satisfy the differential equation

$$
d^{2}\left(\mu^{\varepsilon}\right)_{j}^{( \pm)} / d x_{N}^{2}-\left(\nu_{j}-\lambda-i \varepsilon\right)\left(\mu^{\varepsilon}\right)_{j}^{( \pm)}=0,
$$

and (2) $\mu^{\varepsilon}(x) \in L_{2}(\Omega)$. Lemma 3.1 follows immediately from this. 
Theorem 3.1 (Uniqueness Theorem). Suppose $\lambda \in \Lambda, v_{r}<\lambda<\nu_{r+1}, \mu(x) \in \chi$, and $\bar{A} \mu=\lambda \mu$. Suppose also that conditions (3.2) and (3.3) hold. Then $\mu \equiv 0$.

Proof. We shall show that $\mu(x) \in L_{2}(\Omega)$. This would imply that $\mu \equiv 0$ since $\lambda \in \Lambda$. It follows from (3.2) and (3.3) that the Fourier expansion of $\mu(x)$ and $\partial \mu / \partial x_{N}$ have the form

$$
\begin{aligned}
& \mu(x)= \sum_{j=1}^{r} C_{j}^{+} \exp \left[i\left(\lambda-v_{j}\right)^{1 / 2} x_{N}\right] \eta_{j}(\tilde{x})+\sum_{j=r+1}^{\infty} C_{j}^{+} \exp \left[-\left(\nu_{j}-\lambda\right)^{1 / 2} x_{N}\right] \eta_{j}(\tilde{x}), \\
& \frac{\partial \mu(x)}{\partial x_{N}}=i \sum_{j=1}^{r} C_{j}^{+}\left(\lambda-v_{j}\right)^{1 / 2} \exp \left[i\left(\lambda-v_{j}\right)^{1 / 2} x_{N}\right] \eta_{j}(\tilde{x}) \\
&-\sum_{j=r+1}^{\infty} C_{j}^{+}\left(\nu_{j}-\lambda\right)^{1 / 2} \exp \left[-\left(\nu_{j}-\lambda\right)^{1 / 2} x_{N}\right] \eta_{j}(\tilde{x})
\end{aligned}
$$

for $h^{\prime} \leqq x_{N}$. Similar expansions hold for $x_{N} \leqq-h^{\prime}$. Applying the divergence theorem to the functions $\mu(x)$ and $\bar{\mu}(x)$, and making use of the facts that $\mu=0$ on $\dot{\Omega}$ and $-\Delta \mu=\lambda \mu$ in $\Omega$, we have by integrating over $\Omega_{x_{N}}$

$$
\int_{l_{x_{N}}}\left(\mu \frac{\partial \bar{\mu}}{\partial x_{N}}-\bar{\mu} \frac{\partial \mu}{\partial x_{N}}\right) d \tilde{x}-\int_{l_{-x_{N}}}\left(\mu \frac{\partial \bar{\mu}}{\partial x_{N}}-\bar{\mu} \frac{\partial \mu}{\partial x_{N}}\right) d \tilde{x}=0 \quad \text { for } h^{\prime} \leqq x_{N}
$$

Substituting the Fourier expansions for $\mu(x)$ and $\bar{\mu}(x)$ into (3.6), we obtain:

$$
2 i \sum_{j=1}^{r}\left|C_{j}^{+}\right|^{2}\left(\lambda-v_{j}\right)^{1 / 2}+2 i \sum_{j=1}^{r}\left|C_{j}^{-}\right|^{2}\left(\lambda-v_{j}\right)^{1 / 2}=0 .
$$

Hence $C_{j}^{ \pm}=0, j=1, \ldots, r$. This shows that $\mu(x) \in L_{2}(\Omega)$ and the theorem is proven. Q.E.D.

We next establish the existence of a solution to the boundary value problem (3.1). Set

$$
\mu_{\varepsilon, \nu}(x)=(A-\lambda-\nu-i \varepsilon)^{1 / 2} F(x), \quad \text { where } \varepsilon, \nu>0, \text { and } \lambda \in \Lambda .
$$

The proof of the following existence theorem will be based on certain elliptic estimates, the Fourier expansions of the functions $\mu_{\varepsilon, v}$, and Theorem 3.1.

THEOREM 3.2. Suppose $\lambda \in \Lambda$. Then there exists a function $\mu(x) \in \chi$ such that $\mu_{\varepsilon, v} \longrightarrow \mu$ as $\varepsilon, \nu \rightarrow 0$. $\mu$ satisfies the equation $\bar{A} \mu=\lambda \mu+F$ as well as the conditions (3.2) and (3.3).

Proof. We first show that if $h^{\prime}<\dot{x}_{N}$, then:

$$
\left\|\mu_{\varepsilon, v}\right\|_{\left.0_{i} \dot{x}_{N}\right)} \leqq C \quad \text { uniformly for } \varepsilon, \nu>0 .
$$

Assume that (3.8) does not hold for a particular $\dot{x}_{N}>h^{\prime}$. Then there exists a subsequence $\left\{\mu_{\varepsilon, v}\right\}$ for which $\left\|\mu_{\varepsilon, v}\right\|_{0_{\left(\dot{x}_{N}\right)}} \rightarrow \infty$ as $\varepsilon, \nu \rightarrow 0$. (In arguments of this type we shall denote the members of the subsequence by the same letter as the members 
of the original sequence. Also, we shall denote different constants by the same letter $C$ when there is no danger of confusion.)

Set $\mu_{\varepsilon, v}^{*}=\mu_{\varepsilon, v} /\left\|\mu_{\varepsilon, v}\right\|_{0_{\left(\dot{x}_{N}\right)}}$. Thus we have

$$
\left\|\mu_{\varepsilon, v}^{*}\right\|_{0_{\left(\dot{x}_{N}\right)}}=1
$$

and

$$
\Delta \mu_{\varepsilon, \nu}^{*}=(\lambda+\nu+i \varepsilon) \mu_{\varepsilon, \nu}^{*}+F_{\varepsilon, v}^{*},
$$

where $F_{\varepsilon, v}^{*}=F /\left\|\mu_{\varepsilon, v}\right\|_{0_{\left(\dot{x}_{N}\right)}}$. It is easy to show [3] that for $0<\ddot{x}_{N}<\dot{x}_{N}$, we have

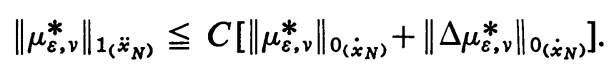

Using (3.9), (3.10), and (3.11), we have

$$
\left\|\mu_{\varepsilon, v}^{*}\right\|_{1_{\left(\ddot{x}_{N}\right)} \leqq C \quad \text { uniformly in } \varepsilon \text { and } \nu .}
$$

By the Rellich selection theorem, we may choose a subsequence $\left\{\mu_{\varepsilon, v}^{*}\right\}$ and a function $\mu^{*}$ defined in $\Omega_{\ddot{x}_{N}}$ satisfying the condition

$$
\left\|\mu_{\varepsilon, \nu}^{*}-\mu^{*}\right\|_{0_{\left(\ddot{x}_{N}\right)}} \rightarrow 0 \quad \text { as } \varepsilon, \nu \rightarrow 0 .
$$

Since $\left\|F_{\varepsilon, v}^{*}\right\|_{0_{(\Omega)}} \rightarrow 0$, we conclude from (3.10), (3.11), and (3.13) that

$$
\left\|\mu_{\varepsilon, v}^{*}-\mu^{*}\right\|_{1\left(\ddot{x}_{N}\right)} \rightarrow 0 \quad \text { for each } 0<\ddot{x}_{N}<\dot{x}_{N} .
$$

We next wish to extend the function $\mu^{*}$ to all of $\Omega$ and show that (3.14) holds for every $0<\ddot{x}_{N}$.

We have the following easily derived inequality

$$
\int_{l x_{N}}\left|\mu_{\varepsilon, v}^{*}\right|^{2} d \tilde{x} \leqq \frac{2}{\left|x_{N}^{1}-x_{N}^{2}\right|}\left\|\mu_{\varepsilon, v}^{*}\right\|_{0_{\left(x_{N}^{1} x_{N}^{2}\right)}^{2}}^{2}+2\left|x_{N}^{1}-x_{N}^{2}\right|\left\|\frac{\partial \mu_{\varepsilon, v}^{*}}{\partial x_{N}}\right\|_{0_{\left(x_{N}^{1} x_{N}^{2}\right)}^{2}}^{2}
$$

where $x_{N}^{1}<x_{N}<x_{N}^{2}, h^{\prime} \leqq x_{N}^{1}$. Thus $\left\{\mu_{\varepsilon, v}^{*}\right\}$ converges in the sense of $L_{2}\left(l_{\ddot{x}_{N}}\right)$ for $h^{\prime} \leqq \ddot{x}_{N}<\dot{x}_{N}$. By Lemma 2.1, we have

$$
\begin{aligned}
\mu_{\varepsilon, v}^{*} & =\sum_{j=1}^{\infty} C_{j}^{+}(\varepsilon, \nu) \exp \left[-\left(\nu_{j}-\lambda-\nu-i \varepsilon\right)^{1 / 2} x_{N}\right] \eta_{j}(\tilde{x}) \\
& =\sum_{j=1}^{\infty} C_{j}^{+^{\prime}}(\varepsilon, \nu) \exp \left[-\left(\nu_{j}-\lambda-\nu-i \varepsilon\right)^{1 / 2}\left(x_{N}-h^{\prime}\right)\right] \eta_{j}(\tilde{x})
\end{aligned}
$$

for $h^{\prime} \leqq x_{N}$, where $\operatorname{Re}\left(\nu_{j}-\lambda-\nu-i \varepsilon\right)^{1 / 2}>0$ for $j=1,2, \ldots$, and $C_{j}^{+^{\prime}}(\varepsilon, \nu)=C_{j}^{+}(\varepsilon, \nu)$ $\cdot \exp \left[-\left(\nu_{j}-\lambda-\nu-i \varepsilon\right)^{1 / 2} h^{\prime}\right]$. Similarly for $x_{N} \leqq-h^{\prime}$. The convergence in the sense of $L_{2}\left(l_{h^{\prime}}\right)$ and $L_{2}\left(l_{-h^{\prime}}\right)$ implies that for each $j$, there exist constants $C_{j}^{( \pm)^{\prime}}$ such that

$$
\sum_{j=1}^{\infty}\left|C_{j}^{( \pm)^{\prime}}(\varepsilon, \nu)-C_{j}^{( \pm)^{\prime}}\right|^{2} \rightarrow 0 \quad \text { as } \varepsilon, \nu \rightarrow 0 .
$$


Also, $\left(\nu_{j}-\lambda-i \varepsilon-\nu\right)^{1 / 2} \rightarrow-i\left(\lambda-v_{j}\right)^{1 / 2}$ for $j \leqq r$ and $\left(\nu_{j}-\lambda-i \varepsilon-\nu\right)^{1 / 2} \rightarrow\left(\nu_{j}-\lambda\right)^{1 / 2}$ for $j>r$ as $\varepsilon, \nu \rightarrow 0$. It thus follows from (3.15) and (3.16) that

$$
\left\|\mu_{\varepsilon, v}^{*}-\mu^{*}\right\|_{1_{\left(x_{N}\right)}} \rightarrow 0
$$

for each $x_{N}$, where outside the domain $\Omega_{h^{\prime}}, \mu^{*}$ is defined as follows

$$
\begin{aligned}
\mu^{*}(x)= & \sum_{j=1}^{r} C_{j}^{+^{\prime}} \exp \left[i\left(\lambda-v_{j}\right)^{1 / 2}\left(x_{N}-h^{\prime}\right)\right] \eta_{j}(\tilde{x}) \\
& +\sum_{j=r+1}^{\infty} C_{j}^{+^{\prime}} \exp \left[-\left(\nu_{j}-\lambda\right)^{1 / 2}\left(x_{N}-h^{\prime}\right)\right] \eta_{j}(\tilde{x}) \text { for } h^{\prime} \leqq x_{N}, \text { and } \\
\mu^{*}(x)= & \sum_{j=1}^{r} C_{j}^{-\prime} \exp \left[-i\left(\lambda-v_{j}\right)^{1 / 2}\left(x_{N}+h^{\prime}\right)\right] \eta_{j}(\tilde{x}) \\
& +\sum_{j=r+1}^{\infty} C_{j}^{-^{\prime}} \exp \left[\left(\nu_{j}-\lambda\right)^{1 / 2}\left(x_{N}+h^{\prime}\right)\right] \eta_{j}(\tilde{x}) \text { for } x_{N} \leqq-h^{\prime} .
\end{aligned}
$$

We now apply the following inequality [10].

$$
\left\|\mu_{\varepsilon, v}^{*}\right\|_{2_{\left(\Omega^{*}\right)}} \leqq C\left[\left\|\mu_{\varepsilon, v}^{*}\right\|_{0_{\left(\Omega^{\prime}\right)}}+\left\|\Delta \mu_{\varepsilon, v}^{*}\right\|_{0_{\left(\Omega^{\prime}\right)}}\right]
$$

where $\Omega^{\prime \prime}$ and $\Omega^{\prime}$ are arbitrary bounded interior subregions of $\Omega$ and $\Omega^{\prime \prime} \subset \Omega^{\prime}$. We thus have $\mu_{\varepsilon, v}^{*} \rightarrow \mu^{*}$. Therefore

$$
\bar{A} \mu^{*}=\lambda \mu^{*} \text {. }
$$

Since $\partial \mu_{\varepsilon, v}^{*} / \partial x_{N}=0$ on $\dot{\Omega}_{x_{N}^{1} x_{N}^{2}}^{\prime}$ for $h^{\prime} \leqq x_{N}^{1}<x_{N}^{2}$, we may apply the preceding arguments to the functions $\partial \mu_{\varepsilon, v}^{*} / \partial x_{N}$ and $\partial \mu^{*} / \partial x_{N}$ to obtain

$$
\begin{aligned}
\frac{\partial \mu^{*}}{\partial x_{N}}= & i \sum_{j=1}^{r} C_{j}^{+}\left(\lambda-v_{j}\right)^{1 / 2} \exp \left[i\left(\lambda-v_{j}\right)^{1 / 2} x_{N}\right] \eta_{j}(\tilde{x}) \\
& -\sum_{j=r+1}^{\infty} C_{j}^{+}\left(\nu_{j}-\lambda\right)^{1 / 2} \exp \left[-\left(\nu_{j}-\lambda\right)^{1 / 2} x_{N}\right] \eta_{j}(\tilde{x}) \quad \text { for } h^{\prime} \leqq x_{N}, \text { and } \\
\frac{\partial \mu^{*}}{\partial x_{N}}= & -i \sum_{j=1}^{r} C_{j}^{-}\left(\lambda-v_{j}\right)^{1 / 2} \exp \left[-i\left(\lambda-\nu_{j}\right)^{1 / 2} x_{N}\right] \eta_{j}(\tilde{x}) \\
& +\sum_{j=r+1}^{\infty} C_{j}^{-}\left(\nu_{j}-\lambda\right)^{1 / 2} \exp \left[\left(\nu_{j}-\lambda\right)^{1 / 2} x_{N}\right] \eta_{j}(\tilde{x}) \text { for } x_{N} \leqq-h^{\prime} .
\end{aligned}
$$

It follows from (3.18)-(3.20) and Theorem 3.1 that $\mu^{*} \equiv 0$. This is impossible since

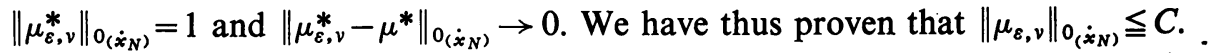

It now follows as before that there exists a subsequence $\left\{\mu_{\varepsilon, v}\right\}$ and a function $\mu(x)$ such that $\mu_{\varepsilon, v} \vec{x} \mu$, and $\mu(x)$ satisfies the conditions (3.2) and (3.3) as well as the equation $\bar{A} \mu=\lambda \mu+F$. Theorem 3.1 implies that the original sequence $\left\{\mu_{\varepsilon, v}\right\}$ converges in the sense of $\chi$ to $\mu(x)$. The theorem is thus proven. Q.E.D.

We may generalize Theorem 3.2 in the following manner. Define $\Lambda_{1}$ to be the set of all complex numbers $\lambda$ in the upper half plane $(\operatorname{Im} \lambda \geqq 0)$ other than the sequences $\left\{\lambda_{n}\right\}$ and $\left\{\nu_{n}\right\}$. Let $\Lambda_{B}$ represent an arbitrary bounded subset of $\Lambda_{1}$. 
Instead of a single function $F(x)$, we consider a sequence of functions $F_{j}(x)$ such that each $F_{j} \in C^{\infty}(\bar{\Omega})$ and $F_{j}(x) \equiv 0$ for $h^{\prime} \leqq\left|x_{N}\right|$ ( $h^{\prime}$ being the same for all $j$ ). For each $\lambda$ in $\Lambda$, let $\mu_{j}(x ; \lambda)$ be the solution of the equation $\bar{A} \mu_{j}=\lambda \mu_{j}+F_{j}$, which satisfies the conditions (3.2) and (3.3). If $\lambda$ is nonreal, set $\mu_{j}(x ; \lambda)=(A-\lambda)^{-1} F_{j}$.

THEOREM 3.3. (a) Suppose $\left\|F_{j}\right\|_{0_{(\Omega)}} \leqq C \quad \forall j=1,2, \ldots$ Then $\left\|\mu_{j}(\cdot ; \lambda)\right\|_{0_{\left(\dot{x}_{N}\right)} \leqq C}$ for each $\dot{x}_{N}$. The constant $C$ is independent of the function $\mu_{j}(x ; \lambda)$ for each $\lambda$ in $\Lambda_{B}$, $j=1,2, \ldots$.

(b) Suppose $\left\|F_{j}-F_{0}\right\|_{0_{(\Omega)}} \rightarrow 0$ and $\lambda^{j} \rightarrow \lambda^{0}$ as $j \rightarrow \infty$, where $\lambda^{j} \in \Lambda_{B}, j=0,1, \ldots$ Then $\left\|\mu_{j}\left(\cdot ; \lambda^{\jmath}\right)-\mu_{0}\left(\cdot ; \lambda^{0}\right)\right\|_{0_{\left(\dot{x}_{N}\right)}} \rightarrow 0$ for each $\dot{x}_{N}$.

Theorem 3.3 can be proven in exactly the same way as Theorem 3.2. We are now in a position to construct the basic tools to be used in our expansion theorem. These are the generalized eigenfunctions of the operator $A$, to be denoted by $w_{n}^{+}(x ; \xi) . w_{n}^{+}(x ; \xi)$ will be expressed in the form $w_{n}^{+}(x ; \xi)=w_{n}^{0}(x ; \xi)+v_{n}^{+}(x ; \xi)$, where $v_{n}^{+}(x ; \xi)$ is to satisfy the conditions (3.2) and (3.3) with $\lambda=\xi^{2}+v_{n}$ (assuming $\left.\lambda \neq v_{j}, j=1,2, \ldots\right) . v_{n}^{+}(x ; \xi)$ will be constructed as a solution to the boundary value problem

$$
\left[-\Delta-\left(\xi^{2}+\nu_{n}\right)\right] v_{n}^{+}(x ; \xi)=0, \quad v_{n}^{+}(x ; \xi)=-w_{n}^{0}(x ; \xi)
$$

on $\dot{\Omega}$. To do this, we use Theorem 3.2 and a standard argument for converting a homogeneous equation with nonzero boundary conditions into an inhomogeneous equation with zero boundary conditions.

Let $\zeta\left(x_{N}\right)$ be a "cutoff" function satisfying the following conditions:

(a) $\zeta\left(x_{N}\right) \in C^{\infty}\left(R^{1}\right)$;

(b) $\zeta\left(x_{N}\right) \equiv 0$ for $h^{\prime} \leqq\left|x_{N}\right|$;

(c) $\zeta\left(x_{N}\right) \equiv 1$ for $\left|x_{N}\right| \leqq h$.

For $x \in \Omega$, set $G_{n}(x ; \xi)=\zeta\left(x_{N}\right) w_{n}^{0}(x ; \xi)$, and $F_{n}(x ; \xi)=\left[-\Delta-\left(\xi^{2}+\nu_{n}\right)\right] G_{n}(x ; \xi)$. Note that

$$
\left\|G_{n}(x ; \xi)\right\|_{0_{(\Omega)}} \leqq C .
$$

Suppose that $\xi^{2}+\nu_{n} \in \Lambda$. By Theorems 3.1 and 3.2, we have a unique solution $\mu_{n}(x ; \xi)$ in $\chi$ of the equation

$$
\bar{A} \mu_{n}(x ; \xi)=\left(\xi^{2}+\nu_{n}\right) \mu_{n}(x ; \xi)+F_{n}(x ; \xi),
$$

such that $\mu_{n}(x ; \xi)$ satisfies (3.2) and (3.3). Set

$$
\begin{aligned}
v_{n}(x ; \xi) & =\mu_{n}(x ; \xi)-G_{n}(x ; \xi), \text { and } \\
w_{n}(x ; \xi) & =w_{n}^{0}(x ; \xi)+v_{n}(x ; \xi) .
\end{aligned}
$$

We thus have $w_{n}(x ; \xi)=0$ on $\dot{\Omega}$, and $\left[\Delta+\left(\xi^{2}+v_{n}\right)\right] w_{n}(x ; \xi)=0$. The functions $w_{n}(x ; \xi)$ will be referred to as distorted plane waves. 
We shall also need the following set of functions. Let $\kappa$ be a complex number such that $\operatorname{Im} \kappa>0$. Set

$$
\begin{aligned}
& F_{n}(x ; \xi ; \kappa)=(-\Delta-\kappa) G_{n}(x ; \xi), \\
& \mu_{n}(x ; \xi ; \kappa)=(A-\kappa)^{-1} F_{n}(x ; \xi ; \kappa), \\
& v_{n}(x ; \xi ; \kappa)=-G_{n}(x ; \xi)+\mu_{n}(x ; \xi ; \kappa),
\end{aligned}
$$

and

$$
w_{n}(x ; \xi ; \kappa)=w_{n}^{0}(x ; \xi)+v_{n}(x ; \xi ; \kappa) .
$$

Set $v_{n}\left(x ; \xi ; \xi^{2}+v_{n}\right)=v_{n}(x ; \xi)$ and $w_{n}\left(x ; \xi ; \xi^{2}+v_{n}\right)=w_{n}(x ; \xi)$ for $\xi^{2}+v_{n} \in \Lambda$.

NoTE. (1) $w_{n}(x ; \xi ; \kappa)=0$ on $\dot{\Omega}$ and satisfies the equation $\left(-\Delta-\xi^{2}-v_{n}\right) w_{n}(x ; \xi ; \kappa)$ $=\left(\kappa-\xi^{2}-v_{n}\right) v_{n}(x ; \xi ; \kappa)$.

(2) It follows from Lemma 3.1 that $v_{n}(x ; \xi ; \kappa)$ dies down exponentially at infinity.

(3) $w_{n}(x ; \xi ; \kappa)$ is an "approximate eigenfunction" for $\kappa$ close to $\xi^{2}+\nu_{n}$ in the sense that $w_{n}(x ; \xi ; \kappa)-w_{n}(x ; \xi) \rightarrow 0$ as $\kappa \rightarrow \xi^{2}+\nu_{n}$. This follows from Theorem 3.3.

THEOREM 3.4. Consider the set of numbers $\kappa$ in $\Lambda_{B}$ such that for each positive integer $j$, we have $\operatorname{Re}\left(\nu_{j}-\kappa\right)^{1 / 2} \neq 0$. We choose that square root for which

$$
\operatorname{Re}\left(\nu_{j}-\kappa\right)^{1 / 2}>0, \quad j=1,2, \ldots
$$

Then

(a) For each multi-index $\alpha=\left(\alpha_{1}, \ldots, \alpha_{N}\right)$, there exists an integer $M_{\alpha}$ and a constant $C_{\alpha}$ such that

$$
\max _{\Omega_{\dot{x}_{N}}}\left|D^{\alpha} v_{n}(x ; \xi ; \kappa)\right| \leqq C_{\alpha}\left(\xi^{2}+v_{n}\right)^{M_{\alpha}},
$$

where $C_{\alpha}$ depends only on $\dot{x}_{N} . D^{\alpha}$ signifies differentiation with respect to $x_{1}, \ldots, x_{N}$.

(b) For each fixed complex number $\kappa$ such that $\operatorname{Im} \kappa>0$, and for each $\alpha=\left(\alpha_{1}, \ldots, \alpha_{n}\right)$ there exist constants $C_{\alpha}$ and $d_{\alpha}>0$ and an integer $M_{\alpha}$ such that for all points $x$ in $\Omega$ for which $x_{N}$ is sufficiently large we have

$$
\left|D^{\alpha} v_{n}(x ; \xi ; \kappa)\right| \leqq\left(\xi^{2}+v_{n}\right)^{M_{\alpha}} C_{\alpha} \exp \left[-d_{\alpha} x_{N}\right]
$$

the constants being independent of $\xi$ and $n$. They will, however, depend on $\kappa$.

Proof. (a) It follows from the theory of elliptic equations [1] that for each $w$ in $\dot{H}_{1}(B)$ where $B$ is a bounded domain with sufficiently smooth boundary we have

$$
\|w\|_{J+2_{(B)}} \leqq C\left[\|\Delta w\|_{J_{(B)}}+\|w\|_{0_{(B)}}\right] \text { provided }\|\Delta w\|_{J_{(B)}}<\infty
$$

$J$ being any nonnegative integer. Using Theorem 3.3, (3.21), and the definitions 
(3.23)-(3.25), it now follows by a simple induction argument that for each $J$, there

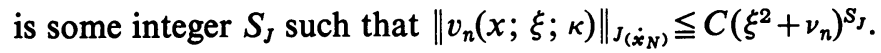

Applying Sobolev's theorem [15], we obtain (3.27). Part (a) is thus proven.

(b) By Lemma 3.1 we have

$$
\begin{aligned}
v_{n}(x ; \xi ; \kappa) & =\sum_{j=1}^{\infty} C_{j}^{+}(\xi, n) \exp \left[-\left(\nu_{j}-\kappa\right)^{1 / 2} x_{N}\right] \eta_{j}(\tilde{x}) & & \text { for } h^{\prime} \leqq x_{N}, \\
& =\sum_{j=1}^{\infty} C_{j}^{-}(\xi, n) \exp \left[\left(\nu_{j}-\kappa\right)^{1 / 2} x_{N}\right] \eta_{j}(\tilde{x}) & & \text { for } x_{N} \leqq-h^{\prime} .
\end{aligned}
$$

Using the expansions (3.29) and taking the integral of $\left|v_{n}(x ; \xi ; \kappa)\right|^{2}$ over $\Omega-\Omega_{h^{\prime}}$, we obtain the following estimate for $c_{j}(\xi, n)$ :

$$
\left|c_{j}(\xi, n)\right| \leqq C\left(\xi^{2}+v_{n}\right) \operatorname{Re}\left(\nu_{j}-\kappa\right)^{1 / 2} \exp \left[\operatorname{Re}\left(\nu_{j}-\kappa\right)^{1 / 2} h^{\prime}\right]
$$

We also have the following inequality

$$
\max _{\tilde{x} \in l}\left|D^{\alpha} \eta_{\jmath}(\tilde{x})\right| \leqq C_{\alpha} \nu_{j}^{|\alpha|+N},
$$

where $C_{\alpha}$ is a constant. Part (b) now follows from (3.29)-(3.31). Q.E.D.

4. The expansion theorem. In $\S 2$, it was shown that the transformation $T_{0}$ given by $T_{0} \psi=\left\{\int_{S} \psi(x) w_{n}^{0}(x ; \xi) d x\right\}$ for each $\psi$ in $C_{0}^{\infty}(S)$, may be used to diagonalize the operator $A_{0}$. In this section, we shall prove an analogous result for the operator $A$.

We first define a transformation $T$ from the dense subset $C_{0}^{\infty}(\Omega)$ of $L_{2}(\Omega)$ into the Hilbert space $H=\bigoplus_{n=1}^{\infty} L_{2}\left(-\infty, \infty, d \sigma_{n}\right)$, where $d \sigma_{n}(\xi)=d \xi$ for each $n$. For every function $\varphi(x)$ in $C_{0}^{\infty}(\Omega)$, set

$$
T \varphi=\left\{\hat{\varphi}_{n}(\xi)\right\}=\left\{\int_{\Omega} \varphi(x) \bar{w}_{n}(x ; \xi) d x\right\}
$$

Denote the eigenvalues (in increasing order) and the corresponding orthonormal eigenfunctions of $A$ by $\lambda_{j}$ and $w_{j}(x)$, respectively. Given any function $f(x)$ in $L_{2}(\Omega)$, set

$$
\hat{f}_{j}=\int_{\Omega} f(x) \bar{w}_{j}(x) d x
$$

Note that it follows from the Fourier expansions of the functions $w_{j}(x)$ that they die down exponentially for $\left|x_{N}\right|$ large.

We now state precisely the main result of this section.

THEOREM 4.1. (i) The transformation $T$ given by (4.1) may be extended to a continuous mapping of $L_{2}(\Omega)$ onto a subspace $\mathscr{S}$ of $H$. For each $f(x)$ in $L_{2}(\Omega)$, we have

$$
T f=\underset{M \uparrow \infty}{\operatorname{li} . \mathrm{m}_{0}}\left\{\int_{\Omega_{M}} f(x) \bar{w}_{n}(x ; \xi) d x\right\}=\left\{\hat{f}_{n}(\xi)\right\}
$$


We also have

$$
\|f\|_{L_{2}(\Omega)}^{2}=\|T f\|_{H}^{2}+\sum_{j=1}^{\infty}\left|\hat{f}_{j}\right|^{2}
$$

(ii) For $f(x)$ in $L_{2}(\Omega)$,

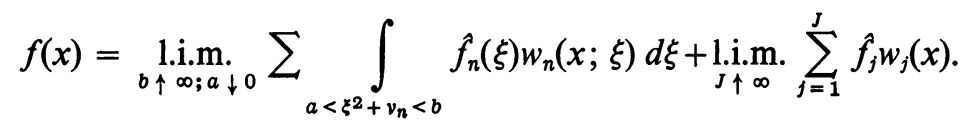

(iii) Given any bounded measurable function $\sigma(\lambda)$, we have for each $f(x)$ in $L_{2}(\Omega)$

$$
\sigma(A) f=\underset{j \uparrow \infty}{\operatorname{li.m} .}\left[\sum \int_{\xi^{2}+v_{n}<J} w_{n}(x ; \xi) \sigma\left(\xi^{2}+v_{n}\right) \hat{f}_{n}(\xi) d \xi+\sum_{j=1}^{J} w_{j}(x) \sigma\left(\lambda_{j}\right) \hat{f}_{j}\right] .
$$

Also, $f(x) \in D(A)$ if and only if

$$
\sum_{n=1}^{\infty} \int_{-\infty}^{\infty}\left|\xi^{2}+\nu_{n}\right|^{2}\left|\hat{f}_{n}(\xi)\right|^{2} d \xi+\sum_{j=1}^{\infty}\left|\lambda_{j}\right|^{2}\left|\hat{f}_{j}\right|^{2}<\infty
$$

In this case, we have

$$
A f=\underset{j \uparrow \infty}{\operatorname{lin} . \mathrm{m}}\left[\sum \int_{\xi^{2}+v_{n}<J} w_{n}(x ; \xi)\left(\xi^{2}+v_{n}\right) \hat{f}_{n}(\xi) d \xi+\sum_{j=1}^{J} w_{j}(x) \lambda_{j} \hat{f}_{j}\right] .
$$

In $\S 5$, we shall prove that the subspace $\mathscr{S}$ of Theorem 4.1 is the entire space $H$. The remainder of this section will be devoted to proving Theorem 4.1. Let $\left\{E_{\lambda}\right\}$ represent the spectral resolution associated with the selfadjoint operator $A$. Thus $E_{\lambda}+=E_{\lambda}$, and $A=\int \lambda d E_{\lambda}$. Theorem 4.1 will follow easily once we have proven the following basic result. Suppose the interval $[a, b]$ is a subset of $\Lambda$. Then

$$
\frac{1}{2}\left(\left(E_{b}+E_{b^{-}}\right) \varphi, \varphi\right)_{(\Omega)}-\frac{1}{2}\left(\left(E_{a}+E_{a^{-}}\right) \varphi, \varphi\right)_{(\Omega)}=\sum \int_{a<\xi^{2}+v_{n}<b}\left|\hat{\varphi}_{n}(\xi)\right|^{2} d \xi,
$$

where $\varphi(x) \in C_{0}^{\infty}(\Omega)$.

To prove (4.6), we use the well-known formula [16]:

$$
\frac{1}{2}\left(\left(E_{b}+E_{b^{-}}\right) f, f\right)_{(\Omega)}-\frac{1}{2}\left(\left(E_{a}+E_{a^{-}}\right) f, f\right)_{(\Omega)}=\operatorname{li.i.m.~}_{\varepsilon \downarrow 0} \frac{\varepsilon}{\pi} \int_{a}^{b}\left\|R_{\tau-i \varepsilon} f\right\|_{L_{2}(\Omega)}^{2} d \tau
$$

for each $f(x)$ in $L_{2}(\Omega)$, where $R_{\tau-i \varepsilon}$ represents the resolvent of $A$, i.e., $R_{\tau-i \varepsilon} f$ $=[A-(\tau-i \varepsilon)]^{-1} f$. Thus, the proof of (4.6) reduces to the evaluation of the limit on the right hand side of (4.7). We begin by obtaining an expression for the integrand $\left\|R_{\tau-i \varepsilon} \varphi\right\|_{L_{2}(\Omega)}^{2}$.

Suppose $\operatorname{Im} \kappa>0, \varphi \in C_{0}^{\infty}(\Omega)$. Define the modified transform $\left\{\hat{\varphi}_{n}(\xi ; \kappa)\right\}$ as follows:

$$
\hat{\varphi}_{n}(\xi ; \kappa)=\int_{\Omega} \varphi(x) \bar{w}_{n}(x ; \xi ; \kappa) d x .
$$

We shall denote by $\left(\hat{\varphi}_{n}(\xi ; \kappa)\right) *$ the complex conjugate of $\left(\hat{\varphi}_{n}(\xi ; \kappa)\right)$. 
Lemma 4.1. Suppose $\varphi(x) \in C_{0}^{\infty}(\Omega)$. Then

(a) $\hat{\varphi}_{n}(\xi ; \kappa)$ is continuous as a function of $\kappa$ for fixed $\xi$ and $n$, where $\kappa \in \Lambda_{1}$.

(b) $\left|\hat{\varphi}_{n}(\xi ; \kappa)\right| \leqq\left(\xi^{2}+v_{n}\right)^{2} C$, where $M_{0}$ is an integer and $C$ is a constant independent of $\xi, n$, and $\kappa$ for $\kappa$ in $\Lambda_{B}$ (a bounded subset of $\Lambda_{1}$ ).

The proof of Lemma 4.1 follows immediately from Theorems 3.3 and 3.4 and the Definition (3.26) of $w_{n}(x ; \xi ; \kappa)$.

TheOREM 4.2. Suppose $\varphi(x) \in C_{0}^{\infty}(\Omega)$. Then

$$
\left\|R_{\bar{\kappa}} \varphi\right\|_{L_{2}(\Omega)}^{2}=\sum_{n=1}^{\infty} \int_{-\infty}^{\infty}\left|\left(\xi^{2}+\nu_{n}-\kappa\right)^{-1} \hat{\varphi}_{n}(\xi ; \kappa)\right|^{2} d \xi
$$

where $\operatorname{Im} \kappa>0$.

Proof. Denote by $\mathscr{D}^{\prime}$ the set of elements $q=\left\{q_{n}(\xi)\right\}$ in $H$ satisfying the conditions (1) $q_{n}(\xi) \equiv 0$ for $n \geqq M$, where $M$ is some positive integer depending on $q$, (2) each $q_{n}(\xi) \in C_{0}^{\infty}\left(R^{2}\right)$, and (3) each $q_{n}(\xi)$ vanishes in a neighborhood of the numbers $\xi$ for which $\xi^{2}+v_{n}=v_{j}$ or $\xi^{2}+v_{n}=\lambda_{j}, j=1,2, \ldots$ Let $\mathscr{D}$ denote the dense subset of $L_{2}(S)$ consisting of functions $\psi(x)$ for which $T^{0} \psi \in \mathscr{D}^{\prime}$. For $\psi(x)$ in $\mathscr{D}$, denote the restriction of $\psi(x)$ to the domain $\Omega$ by $\psi_{\Omega}$. Since $R_{\kappa}$ and $R_{\bar{\kappa}}$ are adjoints, we have:

$$
\left(R_{\bar{\kappa}} \varphi, \psi_{\Omega}\right)_{(\Omega)}=\left(\varphi, R_{\kappa}\left(\psi_{\Omega}\right)\right)_{(\Omega)} .
$$

Now $R_{\kappa}\left(\psi_{\Omega}\right)$ may be explicitly given by the formula

$$
R_{\kappa}\left(\psi_{\Omega}\right)(x)=R_{\psi}(x ; \kappa)=\sum_{n=1}^{\infty} \int_{-\infty}^{\infty} \frac{\hat{\psi}_{n}^{0}(\xi) w_{n}(x ; \xi ; \kappa) d \xi}{\xi^{2}+\nu_{n}-\kappa}
$$

for $x$ in $\Omega$. To see that (4.11) is true, we differentiate the series termwise under the integral sign and observe that $w_{n}(x ; \xi ; \kappa)$ satisfies the differential equation

$$
(-\Delta-\kappa) w_{n}(x ; \xi ; \kappa)=\left(\xi^{2}+v_{n}-\kappa\right) w_{n}^{0}(x ; \xi),
$$

as well as the boundary conditions $w_{n}(x ; \xi ; \kappa)=0$ on $\dot{\Omega}$. Thus

$$
(-\Delta-\kappa) R_{\psi}(x ; \kappa)=\sum_{n=1}^{\infty} \int_{-\infty}^{\infty} \hat{\psi}_{n}^{0}(\xi) w_{n}^{0}(x ; \xi) d \xi=\psi(x) \quad \text { for } x \text { in } \Omega
$$

and

$$
R_{\psi}(x ; \kappa)=0 \quad \text { on } \dot{\Omega} .
$$

It follows immediately from Theorem 3.4 and integration by parts that $R_{\psi}(x ; \kappa)$ and its first and second derivatives are square integrable. We have thus completed the proof of (4.11).

Now substitute (4.11) in equation (4.10) and interchange the order of the 
summation and integrations on the right hand side. (This may be justified using Fubini's theorem.) Thus

$$
\begin{aligned}
\left(R_{\bar{\kappa}} \varphi, \psi_{\Omega}\right)_{(\Omega)} & =\sum_{n=1}^{\infty} \int_{-\infty}^{\infty}\left(\xi^{2}+\nu_{n}-\bar{\kappa}\right)^{-1} \hat{\varphi}_{n}(\xi ; \kappa)\left(\hat{\psi}_{n}^{0}(\xi)\right)^{*} d \xi \\
& =\left(\left(\xi^{2}+\nu_{n}\right)^{-J}\left(\xi^{2}+\nu_{n}-\bar{\kappa}\right)^{-1} \hat{\varphi}_{n}(\xi ; \kappa), \hat{\psi}_{n}^{0}(\xi)\left(\xi^{2}+\nu_{n}\right)^{J}\right)_{H},
\end{aligned}
$$

where $J$ is an integer chosen sufficiently large that

$$
\left\{\left(\xi^{2}+\nu_{n}\right)^{-J}\left(\xi^{2}+\nu_{n}-\bar{\kappa}\right)^{-1} \hat{\varphi}_{n}(\xi ; \kappa)\right\} \in H .
$$

The fact that there exists such a $J$ follows from Lemma 4.1 and the following estimate, [2]:

$$
\sum_{n=1}^{\infty} \frac{1}{\nu_{n}^{J_{0}}}<\infty \quad \text { for some integer } J_{0}
$$

We extend $R_{\bar{\kappa}} \varphi$ to a function $R_{\bar{\kappa}}^{S} \varphi$ defined in all of $S$ as follows:

$$
\begin{aligned}
R_{\bar{\kappa}}^{S} \varphi(x) & =R_{\bar{\kappa}} \varphi(x) & & \text { for } x \text { in } \Omega, \\
& =0 & & \text { for } x \text { in } S-\Omega .
\end{aligned}
$$

Hence

$$
\left(R_{\bar{\kappa}}^{S} \varphi, \psi\right)_{(S)}=\left(\left(\xi^{2}+\nu_{n}\right)^{-J}\left(\xi^{2}+\nu_{n}-\bar{\kappa}\right)^{-1} \hat{\varphi}_{n}(\xi ; \kappa), \hat{\psi}_{n}^{0}(\xi)\left(\xi^{2}+v_{n}\right)^{J}\right)_{H} .
$$

The isometric property of the transformation $T_{0}$ yields

$$
\left(R_{\bar{\kappa}}^{S} \varphi, \psi\right)_{(S)}=\left(\left(\xi^{2}+\nu_{n}\right)^{-J}\left(R_{\bar{\kappa}}^{S} \varphi\right)_{n}^{\hat{0}}(\xi),\left(\xi^{2}+\nu_{n}\right)^{J} \hat{\psi}_{n}^{0}(\xi)\right)_{H} .
$$

The elements $\left\{\left(\xi^{2}+\nu_{n}\right)^{J} \hat{\psi}_{n}^{0}(\xi)\right\}, \psi \in \mathscr{D}$, are clearly dense in $H$. Comparing (4.18) and (4.19), we conclude:

$$
\left(R_{\bar{\kappa}}^{S} \varphi\right)^{\hat{0}}=\left\{\left(\xi^{2}+\nu_{n}-\bar{\kappa}\right)^{-1} \hat{\varphi}_{n}(\xi ; \kappa)\right\} .
$$

Since $R_{\bar{\kappa}}^{S} \varphi \equiv 0$ in $S-\Omega$, equation (4.20) and the unitarity of $T_{0}$ imply

$$
\left\|R_{\bar{\kappa}} \varphi\right\|_{L_{2}(\Omega)}^{2}=\left\|R_{\bar{\kappa}}^{S} \varphi\right\|_{L_{2}(S)}^{2}=\left\|\left(R_{\bar{\kappa}} \varphi\right) \hat{0}\right\|_{H}^{2}=\sum_{n=1}^{\infty} \int_{-\infty}^{\infty}\left|\left(\xi^{2}+\nu_{n}-\bar{\kappa}\right)^{-1} \hat{\varphi}_{n}(\xi ; \kappa)\right|^{2} d \xi .
$$

This completes the proof of the theorem. Q.E.D.

THEOREM 4.3. For each $\varphi(x)$ in $C_{0}^{\infty}(\Omega)$, equation (4.6) holds, i.e.,

where $[a, b] \in \Lambda$.

$$
\frac{1}{2}\left(\left(E_{b}+E_{b}-\right) \varphi, \varphi\right)_{0_{(\Omega)}}-\frac{1}{2}\left(\left(E_{a}+E_{a}-\right) \varphi, \varphi\right)_{0_{(\Omega)}}=\sum \int_{a<\xi^{2}+v_{n}<b}\left|\hat{\varphi}_{n}(\xi)\right|^{2} d \xi,
$$

Proof. Let $\Lambda_{[a, b]}$ represent the set of complex numbers $\kappa=\tau+i \varepsilon$ satisfying the conditions

(1) $a \leqq \tau \leqq b$,

(2) $0<\varepsilon \leqq \varepsilon_{0}$. 
Suppose $\kappa=\tau+i \varepsilon \in \Lambda_{[a, b]}$. Then by Theorem 4.2, we have

$$
\begin{aligned}
\lim _{\varepsilon \downarrow 0} \frac{\varepsilon}{\pi} \int_{a}^{b}\left\|R_{\tau-i \varepsilon} \varphi\right\|_{L_{2}(\Omega)}^{2} d \tau & =\lim _{\varepsilon \downarrow 0} \frac{\varepsilon}{\pi} \int_{a}^{b} \sum_{n=1}^{\infty} \int_{-\infty}^{\infty} \frac{\left|\hat{\varphi}_{n}(\xi ; \kappa)\right|^{2} d \xi d \tau}{\left|\xi^{2}+\nu_{n}-\bar{\kappa}\right|^{2}} \\
& =\lim _{\varepsilon \downarrow 0} \frac{\varepsilon}{\pi} \sum_{n=1}^{\infty} \int_{-\infty}^{\infty} \int_{a}^{b} \frac{\left|\hat{\varphi}_{n}(\xi ; \kappa)\right|^{2} d \tau d \xi}{\left(\xi^{2}+\nu_{n}-\tau\right)^{2}+\varepsilon^{2}}
\end{aligned}
$$

(where we used Fubini's theorem and Lemma 4.1 to interchange the order of the integrations and the summation). It will follow from Lemma 4.2 that

$$
\lim _{\varepsilon \downarrow 0} \frac{\varepsilon}{\pi} \sum \int_{\xi^{2}+v_{n} \geqq b+1} \int_{a}^{b} \frac{\left|\hat{\varphi}_{n}(\xi ; \kappa)\right|^{2} d \tau d \xi}{\left(\xi^{2}+\nu_{n}-\tau\right)^{2}+\varepsilon^{2}}=0 .
$$

Now employing (4.21), we obtain

$$
\lim _{\varepsilon \downarrow 0} \frac{\varepsilon}{\pi} \int_{a}^{b}\left\|R_{\tau-i \varepsilon} \varphi\right\|_{L_{2}(\Omega)}^{2} d \tau=\sum \int_{\xi^{2}+v_{n} \leqq b+1} \lim _{\varepsilon \downarrow 0} \frac{\varepsilon}{\pi} \int_{a}^{b} \frac{\left|\hat{\varphi}_{n}(\xi ; \kappa)\right|^{2} d \tau d \xi}{\left(\xi^{2}+\nu_{n}-\tau\right)^{2}+\varepsilon^{2}}
$$

We next make use of the following result [17]: If $f(\tau, \varepsilon)$ is a continuous function of $\tau$ and $\varepsilon$ for $0 \leqq \varepsilon \leqq \varepsilon_{0}$, and $\alpha \leqq \tau \leqq \beta$, then

$$
\begin{aligned}
\lim _{\varepsilon \downarrow 0} \frac{\varepsilon}{\pi} \int_{\alpha}^{\beta} \frac{f(\tau, \varepsilon) d \tau}{(A-\tau)^{2}+\varepsilon^{2}} & =0 & & \text { if } A<\alpha \text { or } \beta<A, \\
& =f(A, 0) & & \text { if } \alpha<A<\beta .
\end{aligned}
$$

In our case, for each fixed $\xi$ and $n$, set $f(\tau, \varepsilon)=\left|\hat{\varphi}_{n}(\xi ; \tau+i \varepsilon)\right|^{2}$. By Lemma 4.1, $\hat{\varphi}_{n}(\xi ; \kappa)$ is continuous in $\kappa$ for fixed $\xi$ and $n$. Consequently (4.23) yields:

$$
\begin{aligned}
\lim _{\varepsilon \downarrow 0} \frac{\varepsilon}{\pi} \int_{a}^{b} \frac{\left|\hat{\varphi}_{n}(\xi ; \tau+i \varepsilon)\right|^{2} d \tau}{\left(\xi^{2}+\nu_{n}-\tau\right)^{2}+\varepsilon^{2}} & =0 & & \text { if } \xi^{2}+\nu_{n}<a \text { or } \xi^{2}+\nu_{n}>b, \\
& =\left|\hat{\varphi}_{n}(\xi)\right|^{2} & & \text { if } a<\xi^{2}+\nu_{n}<b .
\end{aligned}
$$

We conclude from (4.22) and (4.24):

$$
\lim _{\varepsilon \downarrow 0} \frac{\varepsilon}{\pi} \int_{a}^{b}\left\|R_{\tau-i \varepsilon} \varphi\right\|_{L_{2}(\Omega)}^{2} d \tau=\sum \int_{a<\xi^{2}+v_{n}<b}\left|\hat{\varphi}_{n}(\xi)\right|^{2} d \xi .
$$

Combining (4.25) with (4.7) we see that the theorem will be proven as soon as we establish the equality (4.21).

LEMMA 4.2. For each $\varphi$ in $C_{0}^{\infty}(\Omega)$

$$
\lim _{\varepsilon \downarrow 0} \frac{\varepsilon}{\pi} \sum \int_{\xi^{2}+v_{n} \geqq b+1} \int_{a}^{b} \frac{\left|\hat{\varphi}_{n}(\xi ; \kappa)\right|^{2} d \tau d \xi}{\left(\xi^{2}+\nu_{n}-\tau\right)^{2}+\varepsilon^{2}}=0,
$$

where $\kappa=\tau+i \varepsilon \in \Lambda_{[a, b]}$.

Proof. We shall first prove that

$$
\sum_{n=1}^{\infty} \int_{-\infty}^{\infty} \frac{\left|\hat{\varphi}_{n}^{0}(\xi)-\hat{\varphi}_{n}(\xi ; \kappa)\right|^{2}}{\left(1+\xi^{2}+v_{n}\right)^{2}} d \xi \leqq C,
$$


$C$ being independent of $\kappa$ in $\Lambda_{[a, b]}$. It follows from the definitions of $\hat{\varphi}_{n}^{0}(\xi)$ and $\hat{\varphi}_{n}(\xi, \kappa)$ that

$$
\hat{\varphi}_{n}(\xi ; \kappa)-\hat{\varphi}_{n}^{0}(\xi)=\int_{\Omega} \bar{v}_{n}(x ; \xi ; \kappa) \varphi(x) d x .
$$

Let $\mathscr{G}$ be the set of functions $\psi(x)$ defined in $S$ and satisfying the condition that $A_{0}^{m}(\psi) \in D\left(A_{0}\right)$, i.e., $\Delta^{m} \psi \in L_{2}(S)$ and $\Delta^{m} \psi=0$ on $\dot{S}$ for each $m=0,1, \ldots$ Then

$$
\sum_{n=1}^{\infty} \int_{-\infty}^{\infty} \hat{\psi}_{n}^{0}(\xi)\left[\left(\hat{\varphi}_{n}(\xi ; \kappa)\right)^{*}-\left(\hat{\varphi}_{n}^{0}(\xi)\right)^{*}\right] d \xi=\sum_{n=1}^{\infty} \int_{-\infty}^{\infty} \hat{\psi}_{n}^{0}(\xi)\left[\int_{B} v_{n}(x ; \xi ; \kappa) \bar{\varphi}(x) d x\right] d \xi
$$

where $B=$ support of $\varphi(x)$ and $\psi(x) \in \mathscr{G}$. We may use the fact that

$$
\sum_{n=1}^{\infty} \int_{-\infty}^{\infty}\left|\xi^{2}+\nu_{n}\right|^{j}\left|\hat{\psi}_{n}^{0}(\xi)\right|^{2} d \xi<\infty \quad \text { for } j=1,2, \ldots,
$$

as well as Theorem 3.4 and Fubini's theorem to interchange the order of the integrations and summation on the right hand side. Setting

$$
U_{\psi}(x ; \kappa)=\sum_{n=1}^{\infty} \int_{-\infty}^{\infty} \hat{\psi}_{n}^{0}(\xi) v_{n}(x ; \xi ; \kappa) d \xi
$$

we thus have

$$
\sum_{n=1}^{\infty} \int_{-\infty}^{\infty} \hat{\psi}_{n}^{0}(\xi)\left[\left(\hat{\varphi}_{n}(\xi ; \kappa)\right)^{*}-\left(\hat{\varphi}_{n}^{0}(\xi)\right)^{*}\right] d \xi=\int_{B} \bar{\varphi}(x) U_{\psi}(x ; \kappa) d x .
$$

Therefore

$$
\begin{aligned}
\left|\sum_{n=1}^{\infty} \int_{-\infty}^{\infty} \hat{\psi}_{n}^{0}(\xi)\left[\left(\hat{\varphi}_{n}(\xi ; \kappa)\right)^{*}-\left(\hat{\varphi}_{n}^{0}(\xi)\right)^{*}\right] d \xi\right|^{2} & \\
& \leqq\left(\int_{B}|\varphi(x)|^{2} d x\right)\left(\int_{B}\left|U_{\psi}(x ; \kappa)\right|^{2} d x\right) \\
& \leqq C \int_{B}\left|U_{\psi}(x ; \kappa)\right|^{2} d x,
\end{aligned}
$$

the constant $C$ being independent of $\kappa$ in $\Lambda_{[a, b]}$.

We wish to show that the integral on the right hand side of (4.28) is bounded uniformly with respect to $\kappa$ in $\Lambda_{[a, b]}$. We denote by $G_{\kappa}(x ; y)$, Green's function for the operator $A-\kappa$, where the parameter point $y$ is confined to the set $B$. Now $U_{\psi} \in C^{\infty}(\Omega), U_{\psi}(x ; \kappa)=-\psi(x)$ on $\dot{\Omega}$ and $\psi(x)=0$ on $\dot{\Omega}-\dot{\Omega}_{h}^{\prime}$. Hence for each $y$ in $B$, we have

$$
U_{\Downarrow}(y ; \kappa)=-\int_{\dot{\Omega}_{h}^{\prime}} \psi(x) \frac{\partial G_{\kappa}(x ; y)}{\partial n} d s_{x}
$$

where $s_{x}$ represents arc length along $\dot{\Omega}$, and $n$ represents the outward directed normal on $\dot{\Omega}$. We now prove that

$$
\max _{x \in \dot{\Omega}_{h}^{\prime} ; y \in B}\left|\frac{\partial G_{x}(x ; y)}{\partial n}\right| \leqq \mathrm{C} .
$$


Let $G_{\kappa}^{0}(x ; y)$ denote Green's function for the operator $A_{0}$. It follows from the results in a paper by Tikhonov and Samarskiy $\left({ }^{4}\right)[12]$ that

$$
\max _{x \in \dot{\Omega}_{h^{\prime} ; y \in B}}\left|\frac{\partial G_{x}^{0}(x ; y)}{\partial n}\right| \leqq C,
$$

where $C$ is independent of $\kappa$ for all $\kappa$ in $\Lambda_{[a, b]}$. We shall prove (4.30) by showing that the function $H_{\kappa}(x ; y)=G_{\kappa}(x ; y)-G_{\kappa}^{0}(x ; y)$ satisfies the inequality

$$
\max _{x \in \dot{\Omega}_{h}^{\prime} ; y \in B}\left|\frac{\partial H_{\kappa}(x ; y)}{\partial n}\right| \leqq C,
$$

uniformly in $\kappa$.

We obtain an expression for $H_{\kappa}(x ; y)$ in terms of the functions $v_{n}^{+}(x ; \xi ; \kappa)$ as follows. Let $\Gamma(x)$ be a "cutoff" function satisfying the following conditions:

(a) $\Gamma(x)=0$ in a neighborhood of $B$.

(b) $\Gamma(x)=1$ in a neighborhood of $\dot{\Omega}_{h}^{\prime}$.

(c) $\Gamma(x)=0$ for $\left|x_{N}\right|>h$.

(d) For each $x_{N}$ in $R^{1}$ and $\tilde{x} \in l$, we have $D^{\alpha} \Gamma\left(x_{N}, \tilde{x}\right)=0$, where $D^{\alpha}$ represents any derivative with respect to the variables $x_{1}, \ldots, x_{N-1}$.

It is easily seen that the function $\Gamma(x) G_{x}^{0}(x ; y) \in \mathscr{G}$ for each fixed point $y$ in $B$.

We now consider the function $U_{\Gamma G_{x}^{0}}(x ; y ; \kappa)$. From the definition (4.27) of the function $U_{\psi}(x ; \kappa)$, it follows that $(-\Delta-\kappa) U_{\psi}(x ; \kappa)=0$ in $\Omega$ and $U_{\psi}(x ; \kappa)=-\psi(x)$ on $\dot{\Omega}$. Thus $\left(-\Delta_{x}-\kappa\right) U_{\Gamma G_{\kappa}^{0}}(x ; y ; \kappa)=0$ in $\Omega$ and $U_{\Gamma G_{\kappa}^{0}}(x ; y ; \kappa)=-G_{\kappa}^{0}(x ; y)$ for $x$ on $\dot{\Omega}$, where $\Delta_{x}$ denotes differentiation with respect to the variables $x_{1}, \ldots, x_{N}$. We have thus shown that $H_{\kappa}(x ; y)=U_{\Gamma G_{\kappa}^{0}}(x ; y ; \kappa)$, i.e., $G_{\kappa}(x ; y)=G_{\kappa}^{0}(x ; y)$ $+U_{\Gamma G_{\kappa}^{0}}(x ; y ; \kappa)$. Using the fact that $T_{0}$ is isometric and

$$
\left\|\Delta_{x}^{m}\left(\Gamma(x) G_{x}^{0}(x ; y)\right)\right\|_{L_{2}(S)}^{2} \leqq C, \quad \text { for each } m=0,1, \ldots,
$$

we conclude that

$$
\sum_{n=1}^{\infty} \int_{-\infty}^{\infty}\left|\left(\xi^{2}+\nu_{n}\right)^{m}\left(\Gamma G_{\kappa}^{0}\right)_{n}^{\wedge}(\xi)\right|^{2} d \xi \leqq C
$$

Estimate (4.32), the definition (3.27) of $U_{\psi}(x ; \kappa)$, the properties of $v_{n}(x ; \xi ; \kappa)$ given by Theorem 3.4(a); and the Schwarz inequality imply:

$$
\max _{x \in \dot{\Omega}_{h}^{\prime} ; y \in B}\left|D_{x} U_{\Gamma G_{\kappa}^{0}}(x ; y ; \kappa)\right|=\max _{x \in \dot{\Omega}_{h}^{\prime} ; y \in B}\left|D_{x} H_{\kappa}(x ; y)\right| \leqq C,
$$

the constant $C$ being independent of $\kappa$ in $\Lambda_{[a, b]}$ and $y$ in $B$. The estimate (4.30) now follows immediately.

$\left.{ }^{4}\right)$ Furthermore, explicit representations are given for $G_{\kappa}^{0}(x ; y)$. For example, it is shown that for the case $N=3$,

$$
G_{\kappa}^{0}(x ; y)=\sum_{n=1}^{\infty} \frac{\eta_{n}(\tilde{x}) \eta_{n}(\tilde{y}) \exp \left[-\left(\nu_{n}-\kappa\right)^{1 / 2}\left|x_{N}-y_{N}\right|\right]}{\left(\nu_{n}-\kappa\right)^{1 / 2}} .
$$


Combining (4.28), (4.29), and (4.30), we have:

$$
\left|\sum_{n=1}^{\infty} \int_{-\infty}^{\infty} \hat{\psi}_{n}^{0}(\xi)\left[\left(\hat{\varphi}_{n}(\xi ; \kappa)\right)^{*}-\left(\hat{\varphi}_{n}^{0}(\xi)\right)^{*}\right] d \xi\right|^{2} \leqq C \int_{\dot{\Omega}_{h}^{\prime}}|\psi(x)|^{2} d s_{x^{*}} .
$$

We next employ the following estimates:

$$
\begin{aligned}
\int_{\dot{\Omega}_{h}^{\prime}}|\psi(x)|^{2} d s_{x} & \leqq C\|\psi\|_{1_{(S)}^{2} \leqq C\|(-\Delta+1) \psi\|_{0_{(S)}}^{2}} \\
& =C \sum_{n=1}^{\infty} \int_{-\infty}^{\infty}\left|\left(1+\xi^{2}+v_{n}\right)\right|^{2}\left|\hat{\psi}_{n}^{0}(\xi)\right|^{2} d \xi
\end{aligned}
$$

Hence, for each $\psi(x)$ in $\mathscr{G}$, we have

$$
\begin{aligned}
\left|\sum_{n=1}^{\infty} \int_{-\infty}^{\infty} \hat{\psi}_{n}^{0}(\xi)\left[\left(\hat{\varphi}_{n}(\xi ; \kappa)\right)^{*}-\left(\hat{\varphi}_{n}^{0}(\xi)\right)^{*}\right] d \xi\right|^{2} & \\
& \leqq C \sum_{n=1}^{\infty} \int_{-\infty}^{\infty}\left|\hat{\psi}_{n}^{0}(\xi)\right|^{2}\left(1+\xi^{2}+\nu_{n}\right)^{2} d \xi
\end{aligned}
$$

The constant $C$ depends only on $\varphi$.

Set $Q_{n}(\xi)=\left(1+\xi^{2}+v_{n}\right) \hat{\psi}_{n}^{0}(\xi)$. We may express (4.35) as follows:

$$
\left|\sum_{n=1}^{\infty} \int_{-\infty}^{\infty} \frac{Q_{n}(\xi)\left[\left(\hat{\varphi}_{n}(\xi ; \kappa)\right)^{*}-\left(\hat{\varphi}_{n}^{0}(\xi)\right)^{*}\right] d \xi}{1+\xi^{2}+\nu_{n}}\right|^{2} \leqq C \sum_{n=1}^{\infty} \int_{-\infty}^{\infty}\left|Q_{n}(\xi)\right|^{2} d \xi
$$

Now the set of elements $\left\{Q_{n}(\xi)\right\}=\left\{\left(1+\xi^{2}+\nu_{n}\right) \hat{\psi}_{n}^{0}(\xi)\right\}$ (where $\psi(x)$ may be any function in $\mathscr{G})$ is dense in $H$ since the elements $(-\Delta+1) \psi(x)$ clearly form a dense subset of $L_{2}(S)$ and $T_{0}$ is an isometry. Therefore (4.36) holds for all elements in $H$ and in particular for the elements

$$
\left\{\frac{\left[\hat{\varphi}_{n}(\xi ; \kappa)-\hat{\varphi}_{n}^{0}(\xi)\right]}{\left(1+\xi^{2}+\nu_{n}\right)}\right\}
$$

(It follows from (4.9) that this element belongs to $H$.)

Applying (4.36) to these elements, we have the estimate (4.26). From this it follows immedately that

$$
\sum_{n=1}^{\infty} \int_{-\infty}^{\infty} \frac{\left|\hat{\varphi}_{n}(\xi ; \kappa)\right|^{2} d \xi}{\left(1+\xi^{2}+\nu_{n}\right)^{2}} \leqq C
$$

Since $C$ is independent of $\kappa$ in $\Lambda_{[a, b]}$, we may now conclude that (4.21) holds for each $\varphi(x)$ in $C_{0}^{\infty}(\Omega)$. Q.E.D.

Having completed the proof of Theorem 4.3, we are now in a position to prove the expansion theorem.

Proof of Theorem 4.1. (i) Suppose $\left[a_{0}, b_{0}\right] \in \Lambda$. By Theorem 4.3, we have for each $\varphi(x)$ in $C_{0}^{\infty}(\Omega)$,

$$
\frac{1}{2}\left(\left(E_{b_{0}}+E_{b_{0}^{-}}\right) \varphi, \varphi\right)_{(\Omega)}-\frac{1}{2}\left(\left(E_{a_{0}}+E_{a_{0}^{-}}\right) \varphi, \varphi\right)_{(\Omega)}=\left.\sum_{a_{0}<\xi^{2}+v_{n}<b_{0}} \int_{\hat{\varphi}_{n}}(\xi)\right|^{2} d \xi
$$


From this it follows easily from the spectral theorem [16] that

$$
\left\|\left(E_{b}-E_{a}\right) \varphi\right\|^{2}=\sum \int_{a<\xi^{2}+v_{n}<b}\left|\hat{\varphi}_{n}(\xi)\right|^{2} d \xi+\sum_{a<\lambda_{j} \leqq b}\left|\hat{\varphi}_{j}\right|^{2},
$$

where $[a, b]$ need not belong to $\Lambda$ now. Letting $a \downarrow 0, b \uparrow \infty$, we conclude that

$$
\|\varphi\|_{L_{2}(\Omega)}^{2}=\sum_{n=1}^{\infty} \int_{-\infty}^{\infty}\left|\hat{\varphi}_{n}(\xi)\right|^{2} d \xi+\sum_{j=1}^{\infty}\left|\hat{\varphi}_{j}\right|^{2} .
$$

This shows that the transformation $T$ is continuous on a dense subset of $L_{2}(\Omega)$ and it can be extended to a continuous mapping from $L_{2}(\Omega)$ onto a closed subspace $\mathscr{S}$ of $H$. Given $f(x)$ in $L_{2}(\Omega)$, set $T f=\left\{f_{n}(\xi)\right\}$. The remainder of part (i) now follows easily.

(ii) Suppose $\psi(x) \in C_{0}^{\infty}(\Omega), f(x) \in L_{2}(\Omega)$. It follows from equation (4.37) and the polarization identity that

$$
\left(\left(E_{b_{0}}-E_{a_{0}}\right) f, \psi\right)_{(\Omega)}=\sum \int_{a_{0}<\xi^{2}+v_{n}<b_{0}} \hat{f}_{n}(\xi)\left(\hat{\psi}_{n}(\xi)\right)^{*} d \xi,
$$

where $\left[a_{0}, b_{0}\right] \in \Lambda$. Using the definition of $\hat{\psi}_{n}(\xi)$ and interchanging the order of the integrations and summation, we obtain

$$
\left(\left(E_{b_{0}}-E_{a_{0}}\right) f, \psi\right)_{(\Omega)}=\left(\sum \int_{a<\xi^{2}+v_{n}<b} \hat{f}_{n}(\xi) w_{n}(\cdot ; \xi) d \xi, \psi\right)_{(\Omega)} .
$$

Since this is true for every $\psi(x)$ in $C_{0}^{\infty}(\Omega)$, we have proven the following result:

$$
\left(E_{b_{0}}-E_{a_{0}}\right) f=\sum \int_{a_{0}<\xi^{2}+v_{n}<b_{0}} \hat{f}_{n}(\xi) w_{n}(x ; \xi) d \xi .
$$

It now follows immediately that for any interval $[a, b]$ (not necessarily in $\Lambda$ )

$$
\left(E_{b}-E_{a}\right) f=\sum \int_{a<\xi^{2}+v_{n}<b} \hat{f}_{n}(\xi) w_{n}(x ; \xi) d \xi+\sum_{a<\lambda_{j}<b} \hat{f}_{j} w_{j}(x) .
$$

Letting $a \downarrow 0, b \uparrow \infty$, we see that part (ii) is proven.

(iii) Suppose $\left[a_{0}, b_{0}\right] \in \Lambda$. Let $\chi_{\left[a_{0}, b_{0}\right]}$ denote the characteristic function of the interval $\left[a_{0}, b_{0}\right]$. As a consequence of (4.39), we have for every pair of functions $f(x), q(x)$ in $L_{2}(\Omega)$ :

(4.41) $\quad\left(\left(E_{b_{0}}-E_{a_{0}}\right) f, q\right)_{(\Omega)}=\sum \int_{a_{0}<\xi^{2}+v_{n}<b_{0}} \hat{f}_{n}(\xi)\left(\hat{q}_{n}(\xi)\right)^{*} d \xi=\left(\chi_{\left\lfloor a_{0}, b_{0}\right]} \hat{f}, \hat{q}\right)_{H}$,

where $\chi_{\left[a_{0}, b_{0}\right]} \hat{f}=\left\{\chi_{\left[a_{0}, b_{0}\right]}\left(\xi^{2}+\nu_{n}\right) \hat{f}_{n}(\xi)\right\}$. Let $\chi_{\left[a_{0}, b_{0}\right]}^{(\mathscr{P})}$ denote the orthogonal projection of $\chi_{\left[a_{0}, b_{0}\right]} \hat{f}$ onto the subspace $\mathscr{S}$. Set

$$
\chi_{\left[a_{0}, b_{0}\right]}^{(\mathscr{S})} \hat{f}=\chi_{\left[a_{0}, b_{0}\right]} \hat{f}-\chi_{\left[a_{0}, b_{0}\right]}^{(\mathscr{S})}
$$


Equation (4.41) implies

$$
\left(\left(E_{b_{0}}-E_{a_{0}}\right) f, q\right)_{(\Omega)}=\left(\chi_{\left[a_{0}, b_{0}\right]}^{(\mathscr{S})} \hat{f}, \hat{q}\right)_{H} .
$$

Since $\left[a_{0}, b_{0}\right] \in \Lambda$, we have

$$
\left(\left(E_{b_{0}}-E_{a_{0}}\right) f, w_{j}\right)_{(\Omega)}=\int_{\Omega}\left(E_{b_{0}}-E_{a_{0}}\right) f(x) \bar{w}_{j}(x) d x=0 \quad \text { for } j=1,2, \ldots
$$

It therefore follows from (4.38) and the polarization identity that

$$
\left(\left(E_{b_{0}}-E_{a_{0}}\right) f, q\right)_{(\Omega)}=\left(\left(\left(E_{b_{0}}-E_{a_{0}}\right) f\right)^{\wedge}, \hat{q}\right)_{H} .
$$

A comparison of the last equation with (4.42) shows that

$$
\left(\left(E_{b_{0}}-E_{a_{0}}\right) f\right)^{\wedge}=\chi_{\left[a_{0}, b_{0}\right]}^{(\mathscr{P})} \hat{f} .
$$

As a consequence of (4.37) we have

$$
\begin{aligned}
\left\|\left(E_{b_{0}}-E_{a_{0}}\right) f\right\|_{L_{2}(\Omega)}^{2} & =\left\|\chi_{\left[a_{0}, b_{0}\right]} \hat{f}\right\|_{H}^{2} \\
& =\left\|\chi_{\left[a_{0}, b_{0}\right]}^{(\mathscr{S})} \hat{f}\right\|_{H}^{2}+\left\|\chi_{\left[a_{0}, b_{0}\right]}^{(\mathscr{S}] \perp} \hat{f}\right\|_{H}^{2} .
\end{aligned}
$$

Also

$$
\left\|\left(E_{b_{0}}-E_{a_{0}}\right) f\right\|_{L_{2}(\Omega)}^{2}=\left\|\left(\left(E_{b_{0}}-E_{a_{0}}\right) f\right)^{\wedge}\right\|_{L_{2}(\Omega)}^{2}=\left\|\chi_{\left[a_{0}, b_{0}\right]}^{(\mathscr{P})} \hat{f}\right\|_{H}^{2},
$$

where we used (4.43) and (4.44). Hence $\chi_{\left[a_{0}, b_{0}\right]} \hat{f}=\chi_{\left[a_{0}, b_{0}\right]}^{(\mathscr{P})} \hat{f}$.

We have thus shown that for $f(x)$ in $L_{2}(\Omega)$, and $\left[a_{0}, b_{0}\right]$ in $\Lambda$, the following equation holds:

$$
\left(\left(E_{b_{0}}-E_{a_{0}}\right) f\right)^{\wedge}=\left\{\chi_{\left[a_{0}, b_{0}\right]}\left(\xi^{2}+\nu_{n}\right) \hat{f}_{n}(\xi)\right\} .
$$

The rest of part (iii) now follows directly from the spectral theorem. Q.E.D.

5. The unitarity of $T$. In the preceding section, it was not shown that the subspace $\mathscr{S}$ is all of $H$. This result will now be proven. We begin by proving that a continuous linear transformation $T^{\prime}$ from $H$ into $L_{2}(\Omega)$ may be defined in such a manner that for each $q=\left\{q_{n}(\xi)\right\}$ in $H$ :

$$
T^{\prime} q=\underset{b \uparrow \infty}{\operatorname{li} . \mathrm{m} .} \sum_{\xi^{2}+v_{n}<b} \int_{n} q_{n}(\xi) w_{n}(x ; \xi) d \xi .
$$

Suppose $q=\left\{q_{n}(\xi)\right\} \in \mathscr{D}^{\prime}$ (recall that the set $\mathscr{D}^{\prime}$ was defined in the proof of Theorem 4.2). Set

$$
L_{q}(f)=(q, \hat{f})_{H}=\sum_{n=1}^{\infty} \int_{-\infty}^{\infty} q_{n}(\xi)\left(\int_{\Omega} w_{n}(x ; \xi) \bar{f}(x) d x\right) d \xi
$$

where $f \in C_{0}^{\infty}(\Omega)$. It is easily seen that for each $q$ in $\mathscr{D}, L_{q}(f)$ is a continuous conjugate linear functional on $C_{0}^{\infty}(\Omega)$. Extend it to all of $L_{2}(\Omega)$. $L_{g}(f)$ now represents 
a bounded conjugate linear functional on $L_{2}(\Omega)$. Hence by the Riesz representation theorem there exists a unique function $q^{\prime}(x)$ in $L_{2}(\Omega)$ such that

$$
L_{q}(f)=\left(q^{\prime}, f\right)_{(\Omega)} \text {. }
$$

It follows from this that $T^{\prime} q=q^{\prime}$ for each $q$ in $\mathscr{D}$, where $T^{\prime}$ is defined by (5.1). $\mathscr{D}$ is dense in $H$. It follows from (5.2) that $T^{\prime}$ is continuous on $\mathscr{D}$. Hence we may extend $T^{\prime}$ to all of $H$. Equation (5.1) will now hold for each $q$ in $H$.

Next suppose the operator $A$ has exactly $k$ linearly independent eigenfunctions $(0 \leqq k \leqq \infty)$. Denote by $l_{k}^{2}$ the Hilbert space consisting of sequences of $k$-tuples of complex numbers with the usual inner product. Thus, if $\alpha=\left(\alpha_{1}, \ldots, \alpha_{k}\right) \in l_{k}^{2}$ and $\beta=\left(\beta_{1}, \ldots, \beta_{k}\right) \in l_{k}^{2}$, then $\sum_{j=1}^{k}\left|\alpha_{j}\right|^{2}<\infty, \sum_{j=1}^{k}\left|\beta_{j}\right|^{2}<\infty$, and $(\alpha, \beta)_{l_{k}^{2}}=\sum_{j=1}^{k} \alpha_{j} \bar{\beta}_{j}$. We shall consider the direct sum $\tilde{H}$ of the Hilbert spaces $H$ and $l_{k}^{2}$. For each $\tilde{q}=\left\{\left\{q_{n}(\xi)\right\},\left\{q_{j}\right\}\right\}$ in $\tilde{H}$, set

$$
\tilde{T}^{\prime} q(x)=\underset{M \uparrow}{\operatorname{li} . \mathrm{m} .}\left[\sum_{\xi^{2}+v_{n} \leqq M} \int_{n}(\xi) w_{n}(x ; \xi) d \xi+\sum_{\lambda_{j} \leqq M} q_{j} w_{j}(x)\right] .
$$

$\tilde{T}^{\prime} q \in L_{2}(\Omega)$. For each $f(x)$ in $L_{2}(\Omega)$, set $\tilde{T} f=\left\{\left\{\hat{f}_{n}(\xi)\right\},\left\{\hat{f}_{j}\right\}\right\}$. It follows fromTheorem 4.1 that $\tilde{T}$ is an isometry from $L_{2}(\Omega)$ onto $\tilde{\mathscr{S}}=\mathscr{S} \oplus l_{k}^{2} \subset \tilde{H}$.

TheOREM 5.1. Suppose $\tilde{q} \in \tilde{H}$. Then

$$
\tilde{T} \tilde{T}^{\prime} \tilde{q}=\tilde{q} .
$$

Note. It is an immediate consequence of this theorem that $H=\mathscr{S}$. The following proof is analogous to that given by Ikebe for the operator $-\Delta$ associated with the exterior problem [5].

Proof. Set $\tilde{\gamma}=\left\{\left\{\gamma_{n}(\xi)\right\},\left\{\gamma_{j}\right\}\right\}=\tilde{q}-\tilde{T} \tilde{T}^{\prime} \tilde{q}$. We shall show that $\tilde{\gamma}=0$. Making use of the unitarity of the transformation $T_{1}=T_{0}^{-1}$, it is clearly sufficient to show that

and

$$
0=T_{1} \gamma=\underset{b \uparrow \infty}{\lim . \mathrm{m}} \sum_{\xi^{2}+v_{n} \leqq b} \int_{n}(\xi) w_{n}^{0}(x ; \xi) d \xi,
$$

$$
\gamma_{j}=0, \quad j=1,2, \ldots, k .
$$

We shall begin by establishing the equation

$$
\sum_{\alpha<\xi^{2}+v_{n}<\beta} \gamma_{n}(\xi) w_{n}(x ; \xi) d \xi=0,
$$

where $[\alpha, \beta] \in \Lambda$.

For any point $x$ in $\Omega$; set

and

$$
z_{m}(x)=\sum \int_{\xi^{2}+v_{n} \leqq m} \gamma_{n}(\xi) w_{n}(x ; \xi) d \xi+\sum_{\lambda_{j} \leqq m} \gamma_{j} w_{j}(x),
$$

$$
Z_{m}(x ; \kappa)=\sum \int_{\xi^{2}+v_{n} \leqq m} \frac{\hat{\gamma}_{n}(\xi) w_{n}(x ; \xi)}{\left(\xi^{2}+\nu_{n}-\kappa\right)} d \xi+\sum_{\lambda_{j} \leqq m} \frac{\hat{\gamma}_{j} w_{j}(x)}{\lambda_{j}-\kappa}
$$


where $\kappa=\tau+i \varepsilon, \varepsilon>0$. It is obvious that $(-\Delta-\kappa) Z_{m}(x ; \kappa)=z_{m}(x)$ and $Z_{m}(x ; \kappa)=0$ on $\dot{\Omega}$. Clearly $z_{m}(x)$ and $Z_{m}(x ; \kappa) \in L_{2}(\Omega)$. Thus $Z_{m}(x ; \kappa)=(A-\kappa)^{-1} z_{m}(x)$. As $m \rightarrow \infty, z_{m} \rightarrow \tilde{T}^{\prime} \gamma=\tilde{T}^{\prime} g-\tilde{T}^{\prime} \tilde{T}\left(\tilde{T}^{\prime} g\right)=0$ in $L_{2}(\Omega)$ by Theorem 4.1, part (ii). Since $\left\|Z_{m}(\cdot, \kappa)\right\|_{L_{2}(\Omega)} \leqq C\left\|z_{m}\right\|_{L_{2}(\Omega)}$, we have

$$
Z_{m}(x ; \kappa) \rightarrow 0 \text { in } L_{2}(\Omega) \quad \text { as } m \rightarrow \infty .
$$

Let $D$ be an arbitrary bounded, measurable subset of $\Omega$. Then (5.7) and (5.8) give

$$
\begin{aligned}
0 & =\lim _{m \rightarrow \infty} \int_{D} Z_{m}(x ; \kappa) d x \\
& =\lim _{m \rightarrow \infty} \int_{D} d x\left[\sum \int_{\xi^{2}+\nu_{n} \leqq m} \frac{w_{n}(x ; \xi) \gamma_{n}(\xi) d \xi}{\xi^{2}+\nu_{n}-\kappa}+\sum_{\lambda_{j} \leqq m} \frac{w_{j}(x) \gamma_{j}}{\lambda_{j}-\kappa}\right] \\
& =\sum_{n=1}^{\infty} \int_{-\infty}^{\infty} \frac{\gamma_{n}(\xi) d \xi}{\xi^{2}+\nu_{n}-\kappa} \int_{D} w_{n}(x ; \xi) d x+\sum_{j=1}^{\kappa} \frac{\gamma_{j}}{\lambda_{j}-\kappa} \int_{D} w_{j}(x) d x .
\end{aligned}
$$

Set

$$
p_{n}^{D}(\xi)=\int_{D} \bar{w}_{n}(x ; \xi) d x, \quad p_{j}^{D}=\int_{D} \bar{w}_{j}(x) d x
$$

Clearly $\left\{\left\{p_{n}^{D}(\xi)\right\},\left\{p_{j}^{D}\right\}\right\} \in \tilde{H}$, i.e.

$$
\sum_{n=1}^{\infty} \int_{-\infty}^{\infty}\left|p_{n}^{D}(\xi)\right|^{2} d \xi+\sum_{j=1}^{k}\left|p_{j}^{D}\right|^{2}<\infty
$$

It now follows from (5.9) that

$$
\sum_{n=1}^{\infty} \int_{-\infty}^{\infty} \frac{\gamma_{n}(\xi) \bar{p}_{n}^{D}(\xi)}{\xi^{2}+\nu_{n}-\kappa} d \xi+\sum_{j=1}^{k} \frac{\gamma_{j} \bar{p}_{j}^{D}}{\lambda_{j}-\kappa}=0
$$

Replace $\kappa$ by $\bar{\kappa}$ in (5.10), then subtract, integrate with respect to $\tau$ from $\alpha$ to $\beta$, and take the limit as $\varepsilon \downarrow 0$. Using the dominated convergence theorem, (4.23), and the fact that

$$
\begin{aligned}
& \sum_{n=1}^{\infty} \int_{-\infty}^{\infty}\left|\gamma_{n}(\xi) \bar{p}_{n}(\xi)\right| d \xi+\sum_{j=1}^{k}\left|\gamma_{j} \bar{p}_{j}^{D}\right| \\
& \leqq\left(\sum_{n=1}^{\infty} \int_{-\infty}^{\infty}\left|\gamma_{n}(\xi)\right|^{2} d \xi\right)^{1 / 2}\left(\sum_{n=1}^{\infty} \int_{-\infty}^{\infty}\left|p_{n}^{D}(\xi)\right|^{2}\right)^{1 / 2} \\
&+\left(\sum_{j=1}^{k}\left|\gamma_{j}\right|^{2}\right)^{1 / 2}\left(\sum_{j=1}^{k}\left|\bar{p}_{j}^{D}\right|^{2}\right)^{1 / 2}<\infty
\end{aligned}
$$


we obtain

$$
\begin{aligned}
0= & \lim _{\varepsilon \downarrow 0}\left[\int_{\alpha}^{\beta} d \tau\left(\sum_{n=1}^{\infty} \int_{-\infty}^{\infty} \frac{2 i \varepsilon \gamma_{n}(\xi) \bar{p}_{n}^{D}(\xi) d \xi}{\left(\xi^{2}+\nu_{n}-\tau\right)^{2}+\varepsilon^{2}}\right)+\int_{\alpha}^{\beta} d \tau\left(\sum_{j=1}^{k} \frac{2 i \varepsilon \gamma_{j} \bar{p}_{j}^{D}}{\left(\lambda_{j}-\tau\right)^{2}+\varepsilon^{2}}\right)\right] \\
= & 2 i \sum_{n=1}^{\infty} \int_{-\infty}^{\infty} \gamma_{n}(\xi) \bar{p}_{n}^{D}(\xi) \lim _{\varepsilon \downarrow 0} \int_{\alpha}^{\beta} \frac{\varepsilon d \tau d \xi}{\left(\xi^{2}+\nu_{n}-\tau\right)^{2}+\varepsilon^{2}} \\
& +2 i \sum_{j=1}^{k} \gamma_{j} \bar{p}_{j}^{D} \lim _{\varepsilon \downarrow 0} \int_{\alpha}^{\beta} \frac{\varepsilon d \tau}{\left(\lambda_{j}-\tau\right)^{2}+\varepsilon^{2}} \\
= & 2 \pi i \int_{D} d x\left(\sum_{\alpha<\xi^{2}+v_{n}<\beta} \gamma_{n}(\xi) w_{n}(x ; \xi) d \xi\right)+0,
\end{aligned}
$$

where we used the fact that $\lambda_{j} \notin[\alpha, \beta]$ for $j=1,2, \ldots, k$. Since $D$ is arbitrary, this implies (5.6).

We now prove (5.4). Given any $\rho \geqq \nu_{1},(5.6)$ implies that for $\varepsilon>0$,

$$
\sum \int_{\rho-\varepsilon<\xi^{2}+v_{n}<\rho+\varepsilon} \gamma_{n}(\xi) w_{n}(x ; \xi) d \xi=0,
$$

where $v_{j}, \lambda_{j} \notin[\rho-\varepsilon, \rho+\varepsilon]$ for $j=1,2, \ldots, k$. Substituting $\lambda=v_{n}+\xi^{2}$, and then letting $\varepsilon \downarrow 0$, we conclude

$$
0=\sum_{n=1}^{N_{\rho}} \frac{\gamma_{n}\left(\left(\rho-\nu_{n}\right)^{1 / 2}\right) w_{n}\left(x ;\left(\rho-\nu_{n}\right)^{1 / 2}\right)+\gamma_{n}\left(-\left(\rho-\nu_{n}\right)^{1 / 2}\right) w_{n}\left(x ;-\left(\rho-\nu_{n}\right)^{1 / 2}\right)}{2\left(\rho-\nu_{n}\right)^{1 / 2}}
$$

where $\nu_{N_{\rho}}<\rho<\nu_{N_{\rho}+1} \rho \in \Lambda$, and $x \in \Omega$.

Now set

$$
\mu(x ; \rho)=\sum_{n=1}^{N_{\rho}} \frac{\gamma_{n}\left(\left(\rho-\nu_{n}\right)^{1 / 2}\right) w_{n}^{0}\left(x ;\left(\rho-\nu_{n}\right)^{1 / 2}\right)+\gamma_{n}\left(-\left(\rho-\nu_{n}\right)^{1 / 2}\right) w_{n}^{0}\left(x ;-\left(\rho-\nu_{n}\right)^{1 / 2}\right)}{2\left(\rho-\nu_{n}\right)^{1 / 2}}
$$

for $\nu_{N_{\rho}}<\rho<\nu_{N_{\rho}+1}, \rho$ in $\Lambda$, and $x$ in $S . \mu(x ; \rho) \in C^{2}(S)$ and $\mu(x ; \rho)=0$ on $S$. Also $-\Delta \mu=\rho \mu$. From (5.12) and the definition of $w_{n}\left(x ;\left(\lambda-\nu_{n}\right)^{1 / 2}\right)$ it follows that for all $x$ in $\Omega$

$$
\mu(x ; \rho)=-\sum_{n=1}^{N_{\rho}} \frac{\gamma_{n}\left(\left(\rho-\nu_{n}\right)^{1 / 2}\right) v_{n}\left(x ;\left(\rho-\nu_{n}\right)^{1 / 2}\right)+\gamma_{n}\left(-\left(\rho-\nu_{n}\right)^{1 / 2}\right) v_{n}\left(x ;-\left(\rho-\nu_{n}\right)^{1 / 2}\right)}{2\left(\rho-v_{n}\right)^{1 / 2}} .
$$

Thus $\mu(x ; \rho)$ satisfies the radiation conditions (3.2) and (3.3) for $\left|x_{N}\right|$ sufficiently large. Therefore, by Theorem 3.1, we have

$$
\begin{aligned}
0 & \equiv \mu(x ; \rho) \\
& =\sum_{n=1}^{N \rho} \frac{\left[\gamma_{n}\left(\left(\rho-v_{n}\right)^{1 / 2}\right) w_{n}^{0}\left(x ;\left(\rho-v_{v}\right)^{1 / 2}\right)+\gamma_{n}\left(-\left(\rho-v_{n}\right)^{1 / 2}\right) w_{n}^{0}\left(x ;-\left(\rho-\nu_{n}\right)^{1 / 2}\right)\right]}{2\left(\rho-\nu_{n}\right)^{1 / 2}} .
\end{aligned}
$$


Integrating with respect to $\rho$ from $\nu_{N_{\rho}}$ to $\nu_{N_{\rho}+1}$ gives

(5.14) $0=$

$$
\int_{v_{N_{\rho}}}^{v_{N_{\rho}+1}} \sum_{n=1}^{N \rho} \frac{\left[w_{n}^{0}\left(x ;\left(\rho-v_{n}\right)^{1 / 2}\right) \gamma_{n}\left(\left(\rho-v_{n}\right)^{1 / 2}\right)+w_{n}^{0}\left(x ;-\left(\rho-v_{n}\right)^{1 / 2}\right) \gamma_{n}\left(-\left(\rho-v_{n}\right)^{1 / 2}\right)\right]}{2\left(\rho-v_{n}\right)^{1 / 2}} d \rho
$$

for each positive integer $N_{\rho}$. Finally, letting $N_{\rho} \rightarrow \infty$, we conclude from (5.14) that for each $x$ in $S$, we have

$0=\sum_{n=1}^{\infty} \int_{v_{n}}^{\infty} \frac{\left[w_{n}^{0}\left(x ;\left(\rho-v_{n}\right)^{1 / 2}\right) \gamma_{n}\left(\left(\rho-v_{n}\right)^{1 / 2}\right)+w_{n}^{0}\left(x ;-\left(\rho-v_{n}\right)^{1 / 2}\right) \gamma_{n}\left(-\left(\rho-v_{n}\right)^{1 / 2}\right)\right]}{2\left(\rho-v_{n}\right)^{1 / 2}} d \rho$.

Substituting $\rho=\xi^{2}+\nu_{n}$, we have $0=\sum_{n=1}^{\infty} \int_{-\infty}^{\infty} w_{n}^{0}(x ; \xi) \gamma_{n}(\xi) d \xi$. This gives (5.4). Therefore

$$
\left\{\gamma_{n}(\xi)\right\} \equiv 0
$$

Finally we prove (5.5). Suppose $\lambda_{J}=\lambda_{J+1}=\cdots=\lambda_{J+s}$ is an eigenvalue of $A$ of multiplicity $s+1$. Choose an interval $[\alpha, \beta]$ such that $\lambda_{j} \in[\alpha, \beta]$ for $J \leqq j \leqq J+s$, and $\lambda_{j} \notin[\alpha, \beta]$ for $j<J$ and $j>J+s$. Repeating the argument used to derive (5.11) and employing (5.15) we conclude that $\int_{D} \sum_{i=0}^{s} \gamma_{J+i} w_{J+i}(x) d x=0$. Since $D$ is arbitrary, it follows that $\sum_{i=1}^{s} \gamma_{J+i} w_{J+i}(x) \equiv 0$. Using the fact that $\left\{w_{J+i}(x)\right\}$ $(i=0, \ldots, S)$ are linearly independent, we have proven (5.5). This completes the proof of Theorem 5.1. Q.E.D.

The results of $\S 2$ together with Theorems 4.1 and 5.1 show that the operators $A_{0}$ and $A^{c}$ (the continuous part of the operator $A$ ) are unitarily equivalent. The functions $w_{n}(x ; \xi)$ form a complete, orthogonal set of generalized eigenfunctions of the operator $A^{c}$. Denote the functions $w_{n}(x ; \xi)$ and $v_{n}(x ; \xi)$ by $w_{n}^{+}(x ; \xi)$ and $v_{n}^{+}(x ; \xi)$, respectively. The behavior of $v_{n}^{+}(x ; \xi)$ for $\left|x_{N}\right|$ sufficiently large is described by the "outgoing" radiation conditions (3.2) and (3.3).

We may obtain another complete, orthogonal set of generalized eigenfunctions $w_{n}^{-}(x ; \xi)=w_{n}^{0}(x ; \xi)+v_{n}^{-}(x ; \xi)$ in the same way. To construct the "incoming" solutions $v_{n}^{-}(x ; \xi)$ we would prove a theorem analogous to Theorem 3.2 (in this case we would have $\varepsilon<0)$. The functions $v_{n}^{-}(x ; \xi)$ constructed in this manner have the following Fourier expansions

$$
\begin{array}{rlr}
v_{n}^{-}(x ; \xi) & =\sum_{j=1}^{\infty} d_{j}^{+} \exp \left[-\left(\nu_{j}-\left(\xi^{2}+\nu_{n}\right)\right)^{1 / 2} x_{N}\right] \eta_{j}(\tilde{x}), & \text { for } h^{\prime} \leqq x_{N}, \\
& =\sum_{j=1}^{\infty} d_{j}^{-} \exp \left[\left(v_{j}-\left(\xi^{2}+v_{n}\right)\right)^{1 / 2} x_{N}\right] \eta_{j}(\tilde{x}), & \text { for } x_{N} \leqq-h^{\prime} ; \\
\frac{\partial v_{n}^{-}(x ; \xi)}{\partial x_{N}} & =\sum_{j=1}^{\infty}-d_{j}^{+}\left(\nu_{j}-\left(\xi^{2}+v_{n}\right)\right)^{1 / 2} \exp \left[-\left(\nu_{j}-\left(\xi^{2}+v_{n}\right)\right)^{1 / 2} x_{N}\right] \eta_{j}(\tilde{x}), \\
& =\sum_{j=1}^{\infty} d_{j}^{-}\left(\nu_{j}-\left(\xi^{2}+v_{n}\right)\right)^{1 / 2} \exp \left[\left(\nu_{j}-\left(\xi^{2}+v_{n}\right)\right)^{1 / 2} x_{N}\right] \eta_{j}(\tilde{x}), & \text { for } h^{\prime} \leqq x_{N},
\end{array}
$$

where $d_{j^{\prime}}^{+}, d_{j}^{-}$are constants, $\left(\nu_{j}-\left(\xi^{2}+\nu_{n}\right)\right)^{1 / 2}=i\left(\xi^{2}+\nu_{n}-v_{j}\right)^{1 / 2}$, and $\xi^{2}+\nu_{n} \in \Lambda$. 
6. Remarks concerning a semi-infinite cylinder. Suppose now that the unperturbed domain is the semi-infinite cylinder $S^{\prime}$ described in $\$ 2$. The perturbed domain $\Omega^{\prime}$ may be defined as follows:

(i) $\Omega^{\prime} \subseteq S^{\prime}$,

(ii) $\Omega^{\prime}=S^{\prime}$ for $h \leqq x_{N}$, and

(iii) $\dot{\Omega}^{\prime}$ is a $C^{\infty}$ surface in $R^{N}$.

Denote the operators $A_{S^{\prime}}$ and $A_{\Omega^{\prime}}$ by $A_{0}$ and $A$ respectively.

The functions $w_{n}^{0}(x ; \xi)=(2 / \pi)^{1 / 2} \sin \xi x_{N} \eta_{n}(\tilde{x})$ were shown in $\S 2$ to form a complete, orthogonal set of generalized eigenfunctions for $A_{0}$. Using the same arguments as in $\S 3-5$, we can prove results analogous to Theorem 4.1 and Theorem 5.1. In this case the distorted plane waves $w_{n}^{ \pm}(x ; \xi)$ have the form $w_{n}^{ \pm}(x ; \xi)=w_{n}^{0}(x ; \xi)$ $+v_{n}^{ \pm}(x ; \xi)$, where the functions $v_{n}^{+}(x ; \xi)\left(v_{n}^{-}(x ; \xi)\right)$ satisfy "outgoing" ("incoming") radiation conditions for $h<h^{\prime} \leqq x_{N}$, similar to those given by the Fourier expansions (3.4), (3.5) $((5.16),(5.17))$. It thus follows that the operator $A^{\mathrm{c}}$ is unitarily equivalent to the operator $A_{0}$.

We now state some known results concerning the point eigenvalues of the operator $A$. First of all, there are no point eigenvalues in the interval $\left(-\infty, \nu_{1}\right)$. Hence the spectrum consists entirely of the interval $\left[\nu_{1}, \infty\right)$. This follows from results in a paper by Jones [7]. This does not rule out the possibility of point eigenvalues embedded in the continuous spectrum. There are no point eigenvalues at all, however, when $\Omega^{\prime}$ satisfies the following additional condition:

$$
\cos \varphi(s) \leqq 0 \text { for each point } x(S) \text { on } \dot{\Omega^{\prime}},
$$

where $\varphi(s)$ is the angle between the exterior normal to $\Omega^{\prime}$ and the positive $x_{N}$-axis.

This was first proven by Rellich [11]. In this case it follows from our results that $A$ is unitarily equivalent to $A_{0}$.

7. More general boundary conditions. Let us now consider a different class of boundary conditions of the form

$$
\partial \mu / \partial n=\rho \mu,
$$

where $\rho=\rho\left(x_{1}, \ldots, x_{N-1}\right)$ is a piecewise continuous function, $\rho \leqq 0$, and $n$ is the outward directed normal from the boundary of the domain. The domains under consideration are the infinite cylinder $S$ described in $\$ 2$ and the perturbed infinite cylinder $\Omega$ described in $\S 3$.

We first define precisely an operator $A_{B}^{(\rho)}$ given by $-\Delta$ acting on functions that satisfy the boundary condition (7.1) on the boundary $\dot{B}$ of a domain $B$ in $R^{N}$. Set

$$
\begin{array}{r}
D\left(A_{B}^{(\rho)^{\prime}}\right)=\left\{\varphi(x) \mid \varphi \in C^{1}(B \cup \dot{B}) \cap C^{2}(B), D^{\alpha} \varphi=O\left(|x|^{-j}\right) \text { for } j=1,2, \ldots,\right. \\
\left.|\alpha| \leqq 2 \text { and }|x| \text { large, } \partial \varphi / \partial n-\rho \varphi=0 \text { on } \dot{B}, \Delta \varphi \in L_{2}(B)\right\}
\end{array}
$$

and set $A_{B}^{(\rho)^{\prime}}=-\Delta \varphi$ for $\varphi$ in $D\left(A_{B}^{(\rho)^{\prime}}\right)$. It follows from Green's formula that $A_{B}^{(\rho)^{\prime}}$ is a positive, symmetric operator. We now define $A_{B}^{(\rho)}$ to be the closure of $A_{B}^{(\rho)}$. It is easily seen that $A_{B}^{(\rho)}$ is a positive, selfadjoint operator. 
Set $A_{S}^{(\rho)}=A_{0}^{(\rho)}$, and $A_{\Omega}^{(\rho)}=A^{(\rho)}$. As in $\S 2$, we may exhibit explicitly a complete, orthogonal set of generalized eigenfunctions $w_{n}^{0}(x ; \xi)$ for the operator $A_{0}^{(\rho)}$. Denote by $\eta_{n}(\tilde{x})$ a complete, orthonormal set of eigenfunctions for the operator $A_{l}^{(\rho)}$, with corresponding eigenvalues $\nu_{n}$ ( $l$ being the cross-section of $S$ ). Suppose $\nu_{1} \leqq \nu_{2} \leqq \cdots$. Then $w_{n}^{0}(x ; \xi)=(2 \pi)^{-1 / 2} e^{i \xi x_{N}} \eta_{n}(\tilde{x})$.

We may construct two sets of generalized eigenfunctions $w_{n}^{ \pm}(x ; \xi)$ for the operator $A^{(\rho)}$ exactly as in $\S 3$. Again the key result is the fact that the boundary value problem $(-\Delta-\lambda) \mu=F, \partial \mu / \partial h=\rho \mu$ on $\dot{\Omega}$ has a unique solution subject to the appropriate radiation conditions at infinity, where $F(x) \equiv 0$ for $h<h^{\prime} \leqq\left|x_{N}\right|$ and $F \in C^{\infty}(\bar{\Omega})$. We also assume that $\nu_{r}<\lambda<\nu_{r+1}$ and $\lambda \neq \lambda_{j}$ for $j=1,2, \ldots$, where $\left\{\lambda_{j}\right\}$ represent the eigenvalues of $A^{(\rho)}$. The proof is the same as that of Theorem 3.2.

Thus we may construct two sets of generalized eigenfunctions $w_{n}^{ \pm}(x ; \xi)$ $=w_{n}^{0}(x ; \xi)+v_{n}^{ \pm}(x ; \xi)$, where $v_{n}^{+}(x ; \xi)\left(v_{n}^{-}(x ; \xi)\right)$ may be expanded in Fourier series of the form (3.4), (3.5), ((5.16), (5.17)). Now, denoting the eigenfunctions of $A^{(\rho)}$ by $w_{j}(x)$, we may show that the results of Theorems 4.1 and 5.1 remain true in this case. All of the proofs in $\$ \$ 4$ and 5 go over essentially unchanged.

8. More general domains. Again we shall consider a perturbed infinite cylinder, $\Omega$, in $R^{N}$. (The same arguments would go through for a perturbed semi-infinite cylinder.) In this case, however, we do not stipulate that $\Omega$ be contained in an infinite cylinder. Thus $\Omega$ need only satisfy the following two conditions (see Figure 2).

(1) $\Omega=S$ for $\left|x_{N}\right| \geqq h$,

(2) $\dot{\Omega}$ is a $C^{2}$ surface.

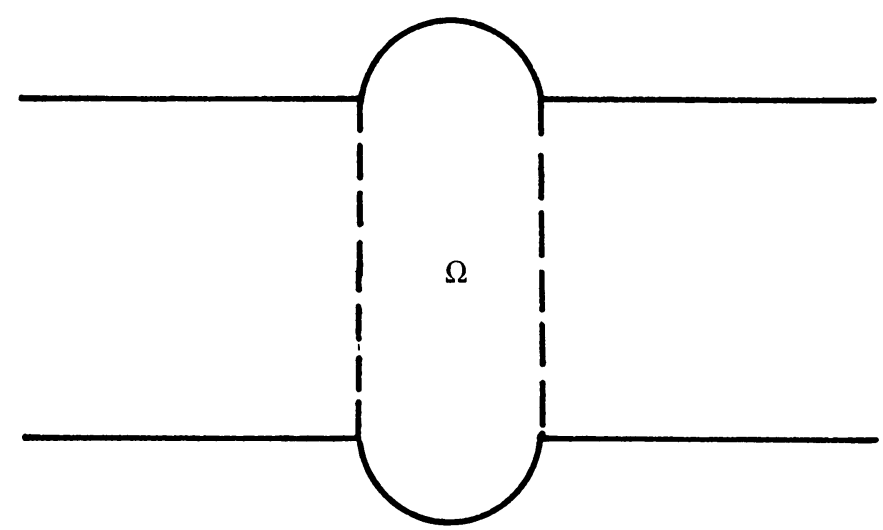

FIGURE 2

We shall show that for $\lambda$ real there can be at most a finite number of bounded linearly independent solutions of the boundary value problem

$$
\begin{aligned}
(\Delta+\lambda) \mu=0 & \text { in } \Omega, \\
\mu=0 & \text { on } \dot{\Omega} .
\end{aligned}
$$


(Actually the result proven in Theorem 8.1 is stronger than that stated above.)

We begin with the following lemma:

LEMMA 8.1. Suppose $\mu(x)$ is a real-valued function defined in $\Omega$ such that $\mu(x) \in L_{2}(\Omega)$ and $\mu(x)$ is a solution of the boundary value problem:

$$
\begin{aligned}
\Delta \mu+a_{1} \mu+a_{2} \partial \mu / \partial x_{N} & =0 & \text { in } \Omega, \\
\mu & =0 & \text { on } \dot{\Omega},
\end{aligned}
$$

where $a_{1}, a_{2}$ are real constants. Then there exists a constant $C$, independent of the function $\mu(x)$ such that

$$
\|\mu\|_{1_{(\Omega)}} \leqq C\|\mu\|_{0_{(\Omega)}} .
$$

Proof. The divergence theorem, combined with (8.3) and (8.4), yields

$$
\begin{aligned}
\int_{\Omega_{r}}|\nabla \mu|^{2} d x & =\sum_{i=1}^{N} \int_{\Omega_{r}}\left|\frac{\partial \mu}{\partial x_{i}}\right|^{2} d x \\
& =\int_{\Omega_{r}} a_{1} \mu^{2}+a_{2} \mu \frac{\partial \mu}{\partial x_{N}} d x+\int_{l_{r}} \mu \frac{\partial \mu}{\partial x_{N}} d \tilde{x}-\int_{l_{-r}} \mu \frac{\partial \mu}{\partial x_{N}} d \tilde{x} .
\end{aligned}
$$

Using the fact that $\left|a_{2} \mu(x) \partial \mu(x) / \partial x_{N}\right| \leqq a_{2}^{2}|\mu(x)|^{2} / 2+\frac{1}{2}\left|\partial \mu(x) / \partial x_{N}\right|^{2}$, we thus have (8.6) $\frac{1}{2} \int_{\Omega_{r}}|\nabla \mu|^{2} d x \leqq \int_{\Omega_{r}}\left(a_{1}+\frac{a_{2}^{2}}{2}\right)|\mu(x)|^{2} d x+\int_{l_{r}} \mu(x) \frac{\partial \mu(x)}{\partial x_{N}} d \tilde{x}-\int_{l_{-r}} \mu(x) \frac{\partial \mu(x)}{\partial x_{N}} d \tilde{x}$.

Set $J_{r}=\int_{l_{r}} \mu(x)\left(\partial \mu(x) / \partial x_{N}\right) d \tilde{x}$. We claim that

$$
\liminf _{r \rightarrow \infty} J_{r} \leqq 0 .
$$

If this were not true, then there would exist constants $r_{0}, C>0$ such that $J_{r} \geqq C$ for $r \geqq r_{0}$. Integrating by parts, we have

$$
\begin{aligned}
\int_{r_{0}}^{r} J_{x_{N}} d x_{N} & =\int_{l} \int_{r_{0}}^{r^{-}} \mu(x) \frac{\partial \mu}{\partial x_{N}} d x_{N} d \tilde{x} \\
& =\int_{l}\left(\left[\mu^{2}(x)\right]_{r_{0}}^{r}-\int_{r_{0}}^{r} \mu(x) \frac{\partial \mu}{\partial x_{N}} d x_{N}\right) d \tilde{x} .
\end{aligned}
$$

This implies that

$$
\int_{r_{0}}^{r} J_{x_{N}} d x=\frac{1}{2} \int_{l}\left[\mu^{2}(\tilde{x}, r)-\mu^{2}\left(\tilde{x}, r_{0}\right)\right] d \tilde{x}
$$

Hence

$$
\begin{aligned}
C\left(r-r_{0}\right) \leqq \int_{r_{0}}^{r} J_{x_{N}} d x_{N} & =\frac{1}{2} \int_{l_{r}} \mu^{2}(x) d \tilde{x}-\int_{l_{r_{0}}} \mu^{2}(x) d \tilde{x} \\
& =C_{0}+\frac{1}{2} \int_{l_{r}} \mu^{2}(x) d \tilde{x} .
\end{aligned}
$$


From this it follows that

$$
\int_{\Omega} \mu^{2}(x) d x=\lim _{r \rightarrow \infty} \int_{\Omega_{r}} \mu^{2}(x) d x=\infty .
$$

This contradicts the fact that $\mu \in L_{2}(\Omega)$. Thus (8.7) holds. Similar reasoning implies

$$
\limsup _{r \rightarrow \infty} J_{-r} \geqq 0 .
$$

We conclude from (8.6)-(8.8) that

$$
\int_{\Omega}|\nabla \mu(x)|^{2} d x \leqq 2\left(a_{1}+\frac{a_{2}^{2}}{2}\right) \int_{\Omega}|\mu(x)|^{2} d x .
$$

This proves the lemma. Q.E.D.

Before proving the main result, we shall define a new Hilbert space $H^{\alpha}$ with norm \langle\rangle$_{\alpha}$. Set $H^{\alpha}=L_{2}\left(\Omega ; d \sigma_{\alpha}(x)\right)$, where $d \sigma_{\alpha}(x)=\exp \left[-\alpha x_{N}\right] d x$, and $\alpha>0$. Thus if $f(x), q(x) \in H^{\alpha}$, then

$$
\begin{aligned}
\langle f, q\rangle_{\alpha} & =\int_{\Omega} f(x) \bar{q}(x) e^{-\alpha x_{N}} d x, \\
\langle f\rangle_{\alpha} & =\left(\int_{\Omega}|f(x)|^{2} e^{-\alpha x_{N}} d x\right)^{1 / 2} .
\end{aligned}
$$

Let $S^{\alpha}$ be the subspace of $H^{\alpha}$ consisting of all solutions of the boundary value problem (8.1), (8.2) ( $\lambda$ real), contained in $H^{\alpha}$. Clearly every bounded solution of (8.1), (8.2) belongs to $S^{\alpha}$ for every $\alpha>0$.

THEOREM 8.1. $S^{\alpha}$ is finite dimensional.

Proof. It is clearly sufficient to prove the result for real-valued functions defined in $\Omega$. Thus we assume that all functions considered are real-valued. We first show that the unit ball in $S^{\alpha}$ is precompact.

Suppose $\left\{\mu_{n}\right\}$ represents a sequence of functions in $S^{\alpha}$ satisfying the condition

$$
\left\langle\mu_{n}\right\rangle_{\alpha}=\left(\int \mu_{n}^{2} \exp \left[-\alpha x_{N}\right] d x\right)^{1 / 2} \leqq 1
$$

We want to show that there exists a subsequence $\left\{\mu_{n,}\right\}$ of $\left\{\mu_{n}\right\}$ such that

$$
\left\langle\mu_{n_{j}}-\mu_{n_{i}}\right\rangle_{\alpha} \rightarrow 0 \quad \text { as } i, j \rightarrow \infty \text {. }
$$

Set

$$
v_{n}(x)=\mu_{n}(x) \exp \left[-(\alpha / 2) x_{N}\right]
$$

It follows from (8.1) and (8.2) that

$$
\begin{aligned}
-\Delta v_{n} & =\lambda v_{n}+\left(\alpha^{2} / 4\right) v_{n}+\alpha\left(\partial v_{n} / \partial x_{N}\right), \\
v_{n} & =0 \quad \text { on } \dot{\Omega} .
\end{aligned}
$$


From (8.10) we have

$$
\left\|v_{n}\right\|_{0_{(\Omega)}} \leqq 1 \quad \text { for } n=1,2, \ldots
$$

(8.13), (8.14), (8.15) and Lemma 8.1 yield

$$
\left\|v_{n}\right\|_{1_{(\Omega)}} \leqq C \quad \text { for } n=1,2, \ldots
$$

We now employ a generalized Phragmén-Lindelöf theorem, due to Lax [8] to conclude that there exists a $\delta>0$ such that

$$
\left\|v_{n}\right\|_{1_{\left(\Omega-\Omega_{M}\right)}} \leqq C e^{-\delta M},
$$

$C$ being independent of $n, M=1,2, \ldots$ For each bounded subdomain $\Omega_{M}$, it follows from the Rellich selection principle and estimate (8.16) that there exists a subsequence $\left\{v_{n_{j}}^{M}\right\}$ such that

$$
\left\|v_{n_{j}}^{M}-v_{n_{i}}^{M}\right\|_{0_{\left(\Omega_{M}\right)}} \rightarrow 0 \quad \text { as } i, j \rightarrow \infty .
$$

The sequences are chosen in such a way that $\left\{v_{n_{j}}^{M}\right\} \subseteq\left\{v_{n_{j}}^{M-1}\right\}$ for each $M$. Denote the diagonal subsequence $\left\{v_{n_{j}}\right\}$ by $\left\{v_{n j}\right\}$. It is obvious that $\left\|v_{n_{j}}-v_{n_{i}}\right\|_{0_{\left(\Omega_{M}\right)}} \rightarrow 0$ as $i, j \rightarrow \infty$, for each $M=1,2, \ldots$

Now, given any $\varepsilon>0$, choose $M_{0}$ so large that

$$
C \exp \left[-\delta M_{0}\right]<\varepsilon / 4 .
$$

Also, choose $i$ and $j$ so large that

$$
\left\|v_{n_{j}}-v_{n_{i}}\right\|_{0_{\left(\Omega_{M_{0}}\right)}}<\varepsilon / 2 .
$$

It follows from (8.17)-(8.19) that

$$
\left\|v_{n_{j}}-v_{n_{i}}\right\|_{0_{(\Omega)}}<\varepsilon \quad \text { for } i \text { and } j \text { sufficiently large. }
$$

From (8.12) and (8.20), we conclude that the subsequence $\left\{\mu_{n j}\right\}$ of $\left\{\mu_{n}\right\}$ is a Cauchy sequence in $H^{\alpha}$. We have thus shown that the unit ball in $S^{\alpha}$ is precompact. This clearly implies that $S^{\alpha}$ is finite dimensional. Q.E.D.

Note that the conclusion of this theorem still holds if the boundary condition (8.2) is replaced by (7.1). The proof is essentially the same.

Acknowledgement. The author wishes to express his thanks to Professor P. D. Lax for suggesting this problem, and for his helpful advice and guidance throughout the preparation of this paper, which is based upon a doctoral dissertation at New York University.

\section{BIBLIOGRAPHY}

1. L. Bers, F. John and M. Schechter, Partial differential equations, Interscience, New York, 1964.

2. R. Courant and D. Hilbert, Methods of mathematical physics, Vol. I, Interscience, New York, 1953.

3. D. M. Eidus, The principle of limiting absorption, Amer. Math. Soc. Transl. 47 (1965), 157-192. 
4. K. Friedrichs, Spectraltheorie halbbeschränkter Operatoren, Math. Ann. 109 (1934), 465-487, 687-713.

5. T. Ikebe, Orthogonality of the eigenfunctions for the exterior problem connected with $-\Delta$, Arch. Rational Mech. Anal. 19 (1965), 71-73.

6. - Eigenfunction expansions associated with the Schroedinger operator and their applications to scattering theory, Arch. Rational Mech. Anal. 5 (1960), 1-34.

7. D. S. Jones, The eigenvalues of $\Delta u+\lambda u=0$ when the boundary conditions are given on semi-infinite domains, Proc. Cambridge Philos. Soc. 49 (1953), 668-684.

8. P. D. Lax, A Phragmén-Lindelöf theorem in harmonic analysis and applications to some questions in the theory of elliptic equations, Comm. Pure Appl. Math. 10 (1957), 361-391.

9. P. D. Lax and R. S. Phillips. Scattering theory, Bull. Amer. Math. Soc. 70 (1964), 130-142.

10. C. Miranda, Equazioni alle derivate parziali di tipo ellittico, Springer, Berlin, 1955.

11. F. Rellich, Uber das asymptotische Verhaltung der Lösungen von $\Delta u+\lambda u=0$ in unendlichen Gebieten, Jber. Deutsch. Math.-Verein. 53 (1943), 157-165.

12. A. A. Samarskiy and A. N. Tikhonov, On excitation of radio wave guides, Akad. Nauk SSSR. Ž. Tehn. Fiz. 17 (1947), 1283-1296.

13. N. Shenk, Eigenfunction expansions and scattering theory for the wave equation in an exterior region, Arch. Rational Mech. Anal. 21 (1966), 120-150.

14. Y. Shizuta, Eigenfunction expansion associated with the operator $-\Delta$ in the exterior domain, Proc. Japan Acad. 39 (1963), 656-660.

15. S. L. Sobolev, Some applications of functional analysis in mathematical physics, Transl. Math. Monographs, Vol. 7, Amer. Math. Soc., Providence, R. I., 1963.

16. M. Stone, Linear transformations in Hilbert space, Amer. Math. Soc. Colloq. Publ., Vol. 15, Amer. Math. Soc., Providence, R. I., 1932.

17. E. Titchmarsh, Introduction to the theory of Fourier integrals, Oxford Univ. Press, London, 1937.

BrookhaVen National Laboratory, LONG ISLAND, New YoRK

NEW YORK UNIVERSITY, New YoRK, New York 